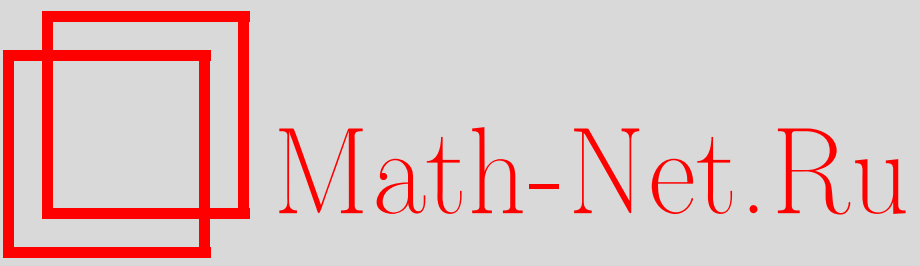

А. Ю. Теплинский, К. М. Ханин, Жесткость для диффеоморфизмов окружности с особенностями, УМН, 2004, том 59, выпуск 2, 137-160

DOI: https://doi.org/10.4213/rm722

Использование Общероссийского математического портала Math-Net.Ru подразумевает, что вы прочитали и согласны с пользовательским соглашением

http://www.mathnet.ru/rus/agreement

Параметры загрузки:

IP: 18.234 .156 .22

26 апреля 2023 г., 06:14:56 


\title{
ЖЕСТКОСТЬ ДЛЯ ДИФФЕОМОРФИЗМОВ \\ ОКРУЖНОСТИ С ОСОБЕННОСТЯМИ
}

\author{
А. Ю. ТЕПЛИНСКИЙ, К. М. ХАнИН
}

\begin{abstract}
В статье обсуждаются недавние резултаты, относящиеся к теории жесткости для диффеоморфизмов окружности с особенностями. Рассматриваются как диффеоморфизмы с изломом, так и критические отображения окружности. В случае излома мы приводим результаты о глобальной гиперболичности ренормализационного оператора, откуда вытекает существование аттрактора типа подковы Смейла. Мы также показываем, что для отображений с особенностями жесткость сильнее, чем в случае диффеоморфизмов, в том смысле, что жесткость не нарушается для нетипичных чисел вращения, которые аномалшно быстро аппроксимируются рациональными. В случае критических поворотов окружности мы доказываем, что любые два таких поворота с одним и тем же порядком особой точки и одинаковым иррациональным числом вращения $C^{1}$-гладко сопряжены между собой.

Библиографияя: 32 названия.
\end{abstract}

\section{СОДЕРЖАНИЕ}

1. Введение

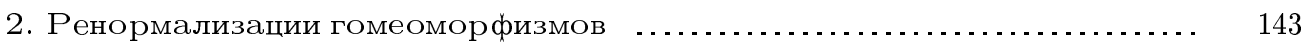

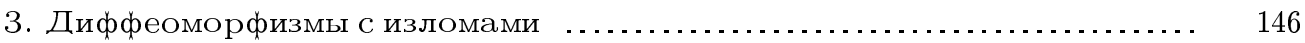

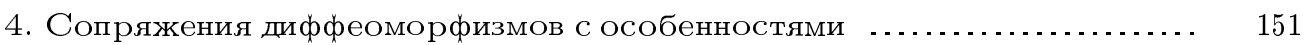

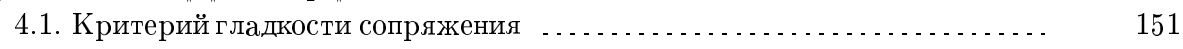

4.2. Условная теорема ............................................. 152

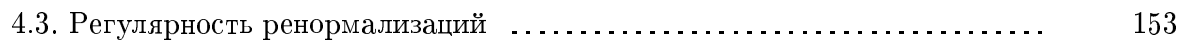

4.4. Главньй этап доказательства ... . . . . . . . . . . . . . . . . . . . . . . 153

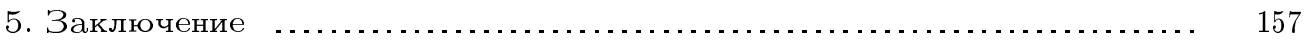

Список литературы . . . . . .

\section{1. Введение}

Настоящая статья основана на докладе, прочитанном одним из авторов (К. М. Ханиным) на конференции, посвященной 100-летию А.Н. Колмогорова. Теория жесткости, о которой пойдет речь, тесно связана с двумя разньми направлениями математической физики и теории динамических систем, в которых Андрею Николаевичу

Работа первого автора выполнена при частичной финансовой поддержке Государственного фонда фундаментальных исследований (ДФФД) Украины (грант № 01.07/00109). 
принадлежат основополагающие результаты. С одной стороны, теория жесткости непосредственно связана с теорией КАМ [1], [2] и, по сушеству, является ее естественньм развитием. С другой стороны, главные технические средства, используемые в теории жесткости, базируются на идеях масштабной инвариантности, которые являются основой революционного вклада Колмогорова в теорию турбулентности [3]-[5]. В этой статье мы надеемся показать, как эти два разных круга идей переплетаются и взаимодействуют в теории жесткости для одномерных отображений.

В самом обшем виде теория жесткости занимается следующей проблемой. Пусть имеются две динамические системы (два отображения), которые гомеоморфино сопряжены друг с другом. Основной вопрос состоит в выяснении того, при каких обстоятельствах сопряжение обязано быть более гладким, т.е. обязано принадлежать классу $C^{1}, C^{k}, k>1$, или $C^{\omega}$. Мы будем рассматривать здесь только одномерные отображения, для которых имеется естественное отношение порядка на фазовом пространстве. В этом случае существование гомеоморфного сопряжения сводится, грубо говоря, к утверждению о том, что для обоих отображений совпадает комбинаторная структура их траекторий. Более того, мы ограничимся рассмотрением лишь монотонных отображений, отвечаюших гомеоморфизмам окружности. Отметим, что хотя монотонность и играет важную роль, мы тем не менее надеемся, что некоторые идеи и результаты этой работы в будушем смогут быть перенесены на более сложньй случай отображений отрезка.

Для сохраняющего ориентацию гомеоморфизма окружности $T$ вся информация о комбинаторной структуре траекторий содержится в его числе врашения $\rho=\rho(T)$ - при условии, что последнее иррационально. При этом если вьполнены некоторые условия регулярности (а именно, $\ln T^{\prime}$ имеет на окружности ограниченную вариацию), то из теории Данжуа вытекает, что диффеоморфизм $T$ с иррациональным числом вращения $\rho$ топологически сопряжен с линейным поворотом окружности на угол $\rho$. Естественным образом возникает вопрос о том, при каких условиях сопряжение $\phi$ является не только непрерьвньп, но и дифференцируемьм. Вопрос этот относится к области теории КАM: когда нелинейньй поворот может быть вьпрямлен посредством гладкой замены координат? Заметим, что $C^{1}$-гладкость является пограничньм случаем между жесткостью и ее отсутствием. Хорошо известно, что $C^{1}$-гладкость сопряжения означает существование у диффеоморфизма $T$ абсолютно непрерьвной инвариантной меры с непрерьвной плотностью. Но для нас более важным будет другое свойство, которое связано с метрическими характеристиками траекторий. А именно, если два гомеоморфизма $T_{1}$ и $T_{2}$ сопряжены между собой $C^{1}$-гладко, то совпадают асимптотические скейлинги вдоль траекторий $T_{1}$ и $T_{2}$. Другими словами, метрические свойства $T_{1}$ и $T_{2}$ асимптотически совпадают.

Впервые результаты о гладкости сопряжений были получены В.И. Арнольдом в 1961 году в его первой работе [6], посвященной теории КАМ. Арнольд доказал, что если число вращения $\rho$ аналитического диффеоморфизма окружности $T$ является диофантовым класса $D_{1}$, а сам диффеоморфизм $T$ близок к линейному, то сопряжение с линейньм поворотом на угол $\rho$ аналитично. Напомним, что число $\rho$ принадлежит классу $D_{\delta}, \delta>0$, если существует $C=C(\rho)>0$ такое, что

$$
\left|\rho-\frac{p}{q}\right| \geqslant \frac{C(\rho)}{q^{2+\delta}}
$$

для всех цельх $p$ и натуральных $q$. Для всякого $\delta>0$ дополнение к множеству $D_{\delta}$ 
имеет нулевую меру Лебега. Арнольдом были также построены контрпримеры, показывающие, что условие типичности (диофантовости) числа $\rho$ является существенньм и теория Данжуа не может быть перенесена на все иррациональные числа вращения. Более точно, Арнольд показал, что в случае чисел вращения, которые могут быть аппроксимированы рациональными аномально быстро, инвариантная мера аналитического диффеоморфизма может быть существенно сингулярной относительно меры Лебега, а при этом и соответствуюшее сопряжение не будет гладким. Арнольд также высказал гипотезу о том, что в одномерном случае локальное условие близости $T$ к линейному повороту не является сушественным для гладкости сопряжения с ним, т.е. должен быть верен аналогичньй глобальньй результат для всех аналитических диффеоморфизмов окружности с диофантовыми числами врашения.

Такой глобальньй результат был впервые получен М.-Р. Эрманом в конце 70-х. Эрман доказал [7], [8], что в случае $T \in C^{k}, k \geqslant 3, \rho \in \bigcap D_{\delta}$ сопряжение $T$ с поворотом на угол $\rho$ гладкое. Позднее теория Эрмана была перенесена Ж.-К. Йоккозом [9] на более широкий класс чисел врашения. Более того, Йоккозу удалось эффективно описать класс чисел врашения, для которых имеется гладкое сопряжение. В конце 80-х Я.Г. Синай и К.М. Ханин [10], [11], а также И. Катцнельсон и Д. Орнштейн [12], [13] показали, что теория может быть построена для диффеоморфизмов класса $C^{2+\alpha}$. Наиболее точньй результат в этой ситуации получен в [11]:

Теорема. Если диффеоморфизм окружности $T$ с числом вращения $\rho \in D_{\delta}$ имеет гладкость $C^{2+\alpha}$, причем $\alpha>\delta>0$, то его сопряжсние с линейным поворотом окружности на угол $\rho$ имеет гладкость $C^{1+\alpha-\delta}$.

Линейные повороты играют выделенную роль в теории жесткости для гладких диффеоморфизмов. Наоборот, в случае диффеоморфизмов с особенностями гладкое сопряжение с линейным поворотом невозможно и основной вопрос состоит в существовании гладкого сопряжения для двух диффеоморфизмов $T_{1}$ и $T_{2}$ с одинаковым типом особых точек. Разумеется, необходимо также потребовать, чтобы числа вращения для обоих отображений были иррациональными и совпадали. Мы будем, в основном, обсуждать ситуацию, в которой как у $T_{1}$, так и у $T_{2}$ есть лишь одна особая точка. Термин “диффеоморфизм с особенностями” используется для того, чтобы подчеркнуть, что всюду, за исключением особой точки (или точек, если их все же несколько), рассматриваемые отображения являются гладкими и обратимыми.

Именно в случае диффеоморфизмов с особенностями идеи масштабной инвариантности или, другими словами, универсальности ренормализационного поведения начинают играть исключительно важную роль. Более формально ренормализации будут определены в следующем разделе, сейчас же мы ограничимся кратким обсуждением основной идеи метода ренорм-групшы. Вместо исходного отображения $T$ изучаются его ренормализации, отвечаюшие специально выбранной последовательности $q_{n}, n \in \mathbb{N}$, моментов времени, в которые траектория отмеченной точки в фазовом пространстве подходит к своему началу особенно близко (обычно речь идет об экспоненциальной по $n$ близости). В каждый из моментов такого "почти возврата" рассматривается ограничение итерации $T^{q_{n}}$ на подходяший (соответственной малости) промежуток вблизи отмеченной точки. Это-то отображение, будучи перенесенньм на единичньй отрезок посредством аффинной замены координат, и назьвают $n$-й ренормализацией исходного. Обычно в качестве отмеченной выбирается особая точка отображения $T$. Основная идея состоит в том, что динамика вблизи особой точки является масштабно инвариантной, т.е. ренормализации двух диффеоморфизмов $T_{1}$ и $T_{2}$ 
асимптотически сближаются, если у последних совпадают типы особенностей и комбинаторика траекторий.

Поскольку последовательные ренормализации могут быть представлены как итерации так называемого "ренормализационного оператора" $\mathscr{R}$, действующего в определенном функциональном пространстве, вопрос о сходимости ренормализаций (под сходимостью мы понимаем их сближение между собой) требует исследования гиперболических свойств оператора $\mathscr{R}$. Говоря коротко, необходимо показать, что два отображения с одинаковьм типом особых точек и одинаковьм комбинаторным типом траекторий лежат на одном устойчивом многообразии оператора $\mathscr{R}$. Впервые метод ренормализаций был использован М. Фейгенбаумом [14], [15], открьвшим универсальные свойства последовательностей бифуркаций удвоения периода для унимодальных отображений отрезка. В этом случае $q_{n}=2^{n}$, а точка максимума является особой (критической). Математическая теория универсальности была построена Д. Салливаном, К. Мак-Малленом и М. Любичем [16]-[18] в конце 80-х - начале 90-х годов. В дальнейшем оказалось, что аналогичная теория может быть развита для диффеоморфизмов окружности с критическими точками, к обсуждению которых мы сейчас приступаем.

Гомеоморфизм $T$ назьвается критическим поворотом окружности $\mathbb{S}^{1}$, если $T \in$ $C^{2+\alpha}\left(\mathbb{S}^{1}\right)$ и $T^{\prime}(x)>0$ всюду за исключением точки $x_{\mathrm{cr}} \in \mathbb{S}^{1}$, в которой $T^{\prime}\left(x_{\mathrm{cr}}\right)=0$. Мы будем говорить, что критическая точка $x_{\mathrm{cr}}$ имеет порядок $1+\beta$, если вблизи нее справедливо асимптотическое представление

$$
T(x)-T\left(x_{\mathrm{cr}}\right)=\left(x-x_{\mathrm{cr}}\right) S\left(\left|x-x_{\mathrm{cr}}\right|^{\beta}\right),
$$

где $S(0)=0, S^{\prime}(0)>0$.

В гладком случае, когда $T \in C^{\infty}\left(\mathbb{S}^{1}\right)$, порядок особой точки отвечает номеру первой не обрашаюшейся в нуль производной. Поскольку отображение $T$ монотонно, то $x_{\mathrm{cr}}$ является точкой перегиба нечетного порядка. Соответственно, $\beta=2 k$, где $k$ - натуральное число. Как уже отмечалось вьше, в этом случае можно построить теорию универсальности, аналогичную теории Салливана-Мак-Маллена-Любича. Это было сделано в работах Э. де Фариа и В. де Мело [19], [20] и М. Ямпольского [21], [22]. Оба эти цикла работ рассматривают действие ренормализационного оператора $\mathscr{R}$ в пространстве $\mathbf{C}$ критических коммутируюших пар и изучают гиперболические свойства $\mathscr{R}$. При этом в некоторых случаях удается доказать, что коммутирующие пары, отвечающие критическим поворотам с заданньм ирращиональным числом вращения, образуют в $\mathbf{C}$ устойчивое многообразие относительно $\mathscr{R}$ и, следовательно, последовательные ренормализации для двух критических поворотов с одинаковьми числами врашения и с одинаковым порядком критических точек сближаются экспоненщиально быстро. Заметим, что для отображения окружности $T$ в качестве моментов почти возврата $q_{n}, n \in \mathbb{N}$, естественно выбрать знаменатели подходящих дробей для числа врашения $\rho(T)$.

$\mathrm{B}[19],[20]$ изучаются критические повороты класса $C^{\infty}$ с $\beta=2 k, k \in \mathbb{N}$, и в случае чисел врашения ограниченного типа доказывается, что ренормализации сближаются экспоненциально быстро. Из этого вытекает, что для любых двух $C^{\infty}$-гладких критических поворотов $T_{1}$ и $T_{2}$ таких, что число $\rho=\rho\left(T_{1}\right)=\rho\left(T_{2}\right)$ имеет ограниченный тип и $\beta=\beta\left(T_{1}\right)=\beta\left(T_{2}\right)=2 k, k \in \mathbb{N}$, сушествует сопряжение гладкости $C^{1+\gamma} \mathrm{c}$ положительньм $\gamma$. Напомним, что иррациональное число назьвается числом ограниченного типа, если ограничена последовательность неполных частных, составляющих его разложение в цепную дробь. 
М. Ямпольский в [22] рассматривает аналитические критические повороты и доказывает, что ренормализации сближаются экспоненциально быстро, для всех иррациональных чисел вращения. Отсюда следует наличие $C^{1+\gamma}$-гладкого сопряжения для несколько более широкого класса чисел врашения. Однако для произвольных иррациональных чисел вращения сама по себе сходимость ренормализаций еще не обеспечивает гладкость сопряжения, что хорошо видно на примере гладких диффеоморфизмов, в случае которых ренормализации сходятся всегда, а сопряжение является гладким лишь при вьполнении определенных диофантовых условий на число вращения. Более того, в [19] построены примеры $C^{\infty}$-гладких критических поворотов, для которых сопряжение не принадлежит классу $C^{1+\gamma}$ ни для какого положительного $\gamma$ несмотря на то, что число врашения $\rho$ является диофантовым (хотя, конечно же, не относится к ограниченному типу). Тем не менее оказьвается, что в случае критических поворотов окружности жесткость намного сильнее, чем в случае гладких диффеоморфизмов. Более точно, справедлива следующая

Теорема 1. Пусть $T_{1}$ и $T_{2}$ - два аналитических критических поворота окружности, для которых $\rho\left(T_{1}\right)=\rho\left(T_{2}\right)=\rho \notin \mathbb{Q} u \beta\left(T_{1}\right)=\beta\left(T_{2}\right)=\beta=2 k, k \in \mathbb{N}$. Тогда между $T_{1}$ и $T_{2}$ существует $C^{1}$-гладкое сопряжение.

Теорема 1 следует из Условной теоремы (теорема 3 в разделе 4), являющейся одним из основных результатов настоящей работы. Условная теорема утверждает, что при выполнении некоторых условий невырожденности сходимость ренормализаций влечет за собой $C^{1}$-гладкость сопряжения. В данное время сходимость ренормализаций для всех иррациональных чисел врашения доказана лишш в случае аналитических поворотов окружности с четными $\beta$, и только с этим связаны соответствуюшие условия в теореме 1. Условие аналитичности является, несомненно, техническим - авторы убеждены в том, что результаты Ямпольского могут быть перенесены на случай $C^{2+\alpha}$-гладких поворотов. Условие на порядок критической точки - более серьезно. Дело в том, что комплексно-аналитические методы, используемые при доказательстве сходимости ренормализаций, исключительно чувствительны по отношению к типу особенности. И все же, никто из специалистов в данной области не сомневается в том, что экспоненциальная сходимость ренормализаций верна для любого действительного $\beta>0$. В этой связи Условная теорема дает веские основания для следуюшей гипотезы.

ГИПОТЕЗА 1. Пусть $T_{1}$ и $T_{2}$ - два критических поворота окружности класса гладкости $C^{2+\alpha}$, для которых $\rho\left(T_{1}\right)=\rho\left(T_{2}\right)=\rho \notin \mathbb{Q} u \beta\left(T_{1}\right)=\beta\left(T_{2}\right)=\beta>0$. Тогда между $T_{1}$ и $T_{2}$ существует $C^{1}$-гладкое сопряжсение.

Заметим, что в самое последнее время Д. Хмелев и М. Ямпольский [23] доказали в случае аналитических критических поворотов локальную $C^{1+\gamma}$-гладкость сопряжения в критической точке. Этот результат позволяет предположить, что в аналитическом случае сопряжение является глобально $C^{1+\gamma}$-гладким. Условие аналитичности здесь сушественно, поскольку, как показывают упомянутые примеры из [19], гладкости $C^{\infty}$ для такого результата недостаточно.

Обратимся теперь к совершенно иному типу особенности, а именно - рассмотрим диффеоморфизмы окружности с изломами. Гомеоморфизм окружности $T$ назьвается диффеоморфизмом с изломом, если принадлежит классу гладкости $C^{2+\alpha}$ всюду за исключением точки $x_{\mathrm{br}} \in \mathbb{S}^{1}$, в которой имеется скачок первой производной: $T^{\prime}\left(x_{\mathrm{br}}-\right) \neq T^{\prime}\left(x_{\mathrm{br}}+\right)$. Дополнительно предполагается, что производная $T^{\prime}(x)$ всюду 
положительна и отделена от нуля. При этом величиной излома назьвается число

$$
c=\sqrt{\frac{T^{\prime}\left(x_{\mathrm{br}}-\right)}{T^{\prime}\left(x_{\mathrm{br}}+\right)}} .
$$

Параметр $c$ играет ту же роль, что и порядок критической точки $1+\beta$, т.е. он определяет тип особенности. При этом имеет смысл сопрягать только диффеоморфизмы с одинаковым значением $c$. Заметим, что диффеоморфизмы с изломом можно рассматривать как однопараметрическое расширение пространства гладких диффеоморфизмов, отвечающих специальному значению параметра $c=1$.

Впервые отображения с особенностями типа излома были рассмотрены Е. Б. Вул и К. М. Ханиным [24], [25]. Ими было показано, что, несмотря на отсутствие критических точек, диффеоморфизмы с изломами проявляют сильно выраженные критические свойства; в частности, имеется нетривиальное ренормгрупповое поведение со сходимостью ренормализаций к дробно-линейньм отображениям. Позднее А. А. Джалилов и К.М. Ханин [26] доказали сингулярность инвариантной меры в случае, когда число врашения $\rho$ иррационально. Теория жесткости для диффеоморфизмов с изломами была построена К. М. Ханиным и Д. Хмелевым [27], [28] для иррациональных $\rho$, разложения которых в цепную дробь периодичны, начиная с некоторой позиции, т.е. для квадратичных иррациональностей. Как и в случае критических поворотов, ключевую роль здесь играет асимптотическое поведение ренормализаций. При этом числа врашения с периодическим разложением в цепную дробь отвечают периодическим орбитам для ренормализационного оператора в том смысле, что ренормализации сходятся к таким периодическим орбитам. Отсюда вытекает утверждение о жесткости. А именно: любые два диффеоморфизма с изломами одинаковой величины $C^{1+\gamma}$-гладко сопряжены друг с другом, если их общее число врашения $\rho$ является квадратичной иррациональностью.

В настоящей работе мы распространяем теорию жесткости на гораздо более обший класс чисел врашения. В качестве первого шага мы доказываем равномерную гиперболичность ренормализационного оператора $\mathscr{R}$ для случая диффеоморфизмов с изломами. Мы также показьваем, что оператор $\mathscr{R}$ обладает неожиданными свойствами симметрии, и достаточно явно строим полную подкову Смейла, отвечающую иррациональным числам вращения. Гипотеза о сушествовании подковы Смейла для критических поворотов окружности, известная как программа Ланфорда, была сформулирована О. Ланфордом в конце 80-х [29]. Замечательно, что в случае диффеоморфизмов с изломами эту программу можно достаточно наглядно провести до конца. В результате удается доказать экспоненциальную сходимость ренормализаций для всех чисел врашения. Отсюда сразу вытекает утверждение о $C^{1+\gamma}$-гладкости сопряжений для чисел вращения ограниченного типа. Однако Условная теорема, о которой мы говорили вьше, позволяет доказать гораздо больше. Пусть $\rho=\left[k_{1}, k_{2}, \ldots\right]$ есть разложение числа вращения в цепную дробь. Определим $M_{\mathrm{o}}$ и $M_{\mathrm{e}}$ как классы чисел вращения, у которых ограничены подпоследовательности неполных частных с нечетными и четными индексами соответственно:

$$
\begin{aligned}
& M_{\mathrm{o}}=\left\{\rho:(\exists C>0)(\forall n \in \mathbb{N}) k_{2 n-1} \leqslant C\right\}, \\
& M_{\mathrm{e}}=\left\{\rho:(\exists C>0)(\forall n \in \mathbb{N}) k_{2 n} \leqslant C\right\} .
\end{aligned}
$$


Теорема 2. Пусть $T_{1}$ и $T_{2}$ - два диффеоморфизма окружности с изломом, причем величины изломов и числа вращения у них совпадают: $c\left(T_{1}\right)=c\left(T_{2}\right)=c$, $\rho\left(T_{1}\right)=\rho\left(T_{2}\right)=\rho \notin \mathbb{Q}$. Тогда междуу $T_{1}$ и $T_{2}$ cуществует $C^{1}-$ гладкое сопряжение, если $c>1$ и $\rho \in M_{\mathrm{o}}$ или же $c<1$ и $\rho \in M_{\mathrm{e}}$.

В этой работе мы ограничиваемся обсуждением основных идей, используемых при доказательстве теорем 1,2 . Полные доказательства всех представленных результатов будут изложены в статьях [30], [31].

В заключение - несколько слов о структуре статьи. В разделе 2 мы описываем обшую конструкцию ренормализаций для гомеоморфизмов окружности. Гиперболические и симметрийные свойства ренормализационного оператора для диффеоморфизмов с изломами изучаются в разделе 3 . Теоремы 1 и 2 вытекают из Условной теоремы, которую мы формулируем в разделе 4 , и там же обсуждается ее доказательство, основанное на анализе динамики итераций вблизи почти параболических точек. В заключительном разделе 5 мы кратко перечисляем полученные результаты и формулируем гипотезу о жесткости для диффеоморфизмов окружности с несколькими особыми точками.

\section{2. Ренормализации гомеоморфизмов}

Пусть $T: \mathbb{S}^{1} \rightarrow \mathbb{S}^{1}$ является произвольным сохраняющим ориентацию гомеоморфизмом единичной окружности $\mathbb{S}^{1}=\mathbb{R} / \mathbb{Z}$. Обозначим через $\pi$ естественную проекцию $\pi: \mathbb{R} \rightarrow \mathbb{S}^{1}$. Поднятием гомеоморфизма $T$ называется гомеоморфизм $L: \mathbb{R} \rightarrow \mathbb{R}$ такой, что $\pi \circ L=T \circ \pi$. Заметим, что поднятие $L$ определено однозначно с точностью до произвольной адлитивной целочисленной константы. Из теоремы Пуанкаре следует, что для каждого $L$ существует предел

$$
\rho(L)=\lim _{i \rightarrow \infty} \frac{y_{i}}{i}
$$

причем не зависяший от начальной точки $y_{0}$ траектории $y_{i}=L^{i} y_{0}, i \geqslant 0$. Числом вращения для гомеоморфизма $T$ назьвается класс эквивалентности для $\rho(L)$ относительно проекции $\pi: \rho(T)=\pi \rho(L)$. Очевидно, что $\rho(T) \in \mathbb{S}^{1}$ и не зависит от выбора поднятия $L$. Нам будет удобно в дальнейшем рассматривать отрезок $[0,1]$ с отождествленными концами как реализацию единичной окружности $\mathbb{S}^{1}$.

Для построения ренормализаций нам понадобится разложение числа вращения в цепную дробь. Напомним, что цепная дробь определяется конечной или бесконечной последовательностью натуральных чисел $k_{n}$, назьваемых неполнылми частны.ми:

$$
\rho=\left[k_{1}, k_{2}, \ldots, k_{n}, \ldots\right]=\frac{1}{k_{1}+\frac{1}{k_{2}+\frac{1}{\frac{\ddots}{k_{n}+\frac{1}{\ddots}}}}} \in[0,1] .
$$

При этом последовательности конечной длины отвечают рациональным числам вращения, а бесконечные - иррациональным. Кроме того, будем считать, что нулевому числу врашения отвечает цепная дробь длины нуль, или, другими словами, пустая последовательность неполных частных. 
Для любого числа $\rho \in(0,1)$ последовательность неполных частных определяется индуктивно с помошњю преобразования Гаусса

$$
\mathscr{G}: \rho \rightarrow\left\{\frac{1}{\rho}\right\}=\frac{1}{\rho}-\left[\frac{1}{\rho}\right],
$$

где для действительного числа $x$ запись $\{x\}$ обозначает его дробную, а $[x]-$ целую часть. Пусть $\rho_{i}=\mathscr{G}^{i} \rho, i \geqslant 0$. В случае рационального $\rho$ последовательность обрывается, когда $\rho_{i}$ обрашается в нуль. Последовательность неполных частных определяется цельми частями в формуле (1), а именно, $k_{i}=\left[1 / \rho_{i-1}\right], i \geqslant 1$. Заметим, что при таком подходе в случае рациональных чисел последнее неполное частное всегда оказьвается больше 1. Хотя разложение в цепную дробь определяется единственным образом, для каждого рационального числа вращения существует два способа его записи в виде конечной цепной дроби: $p / q=\left[k_{1}, \ldots, k_{m}\right]=\left[k_{1}, \ldots, k_{m-1}, k_{m}-1,1\right]$, где $k_{m} \geqslant 2$. Разложение в цепную дробь позволяет найти последовательность рациональных приближений, задаваемых подходящими дробями $p_{n} / q_{n}=\left[k_{1}, \ldots, k_{n}\right]$. Хорошо известно, что числители и знаменатели подходяших дробей удовлетворяют рекуррентным соотношениям: $p_{n+1}=k_{n+1} p_{n}+p_{n-1}, q_{n+1}=k_{n+1} q_{n}+q_{n-1}, n \geqslant 0$. При этом полагают $p_{-1}=1, q_{-1}=0 ; p_{0}=0, q_{0}=1$. В дальнейшем мы рассматриваем цепные дроби только для иррациональных чисел вращения, так что все описываемые ниже конструкции будут определены для сколь угодно больших значений $n$. Для рациональных чисел врашения те же построения корректны, но лишь на протяжении конечного числа шагов.

Возврашаясь к гомеоморфизмам окружности, заметим, что для любой траектории $\xi_{i}=T^{i} \xi_{0}, i \geqslant 0$, последовательность знаменателей подходяших дробей $q_{n}$ определяет моменты почти возвращения, т.е. точки $\xi_{q_{n}}$ приближаются к начальной точке $\xi_{0}$ в следуюшем порядке:

$$
\xi_{q_{1}}<\xi_{q_{3}}<\cdots<\xi_{q_{2 m+1}}<\cdots<\xi_{0}<\cdots<\xi_{q_{2 m}}<\cdots<\xi_{q_{4}}<\xi_{q_{2}}
$$

Определим $n$-й ренормализачионный промежуток $\Delta_{0}^{(n)}$ как дугу $\left[\xi_{0}, \xi_{q_{n}}\right]$ для четного $n$ и $\left[\xi_{q_{n}}, \xi_{0}\right]$ для нечетного. Легко убедиться, что для всякого $n$ первой точкой рассмотренной траектории, попадающей во внутренность отрезка $\Delta_{0}^{(n)}$, является $\xi_{q_{n}+q_{n+1}}$.

Пусть $\Delta_{*}^{(n)}=\Delta_{0}^{(n-1)} \cup \Delta_{0}^{(n)}$. Итерации $T^{q_{n}}$ и $T^{q_{n-1}}$, ограниченные на $\Delta_{0}^{(n-1)}$ и $\Delta_{0}^{(n)}$ соответственно, суть не что иное, как две непрерьвные компоненты функции первого возврашения отображения $T$ на отрезок $\Delta_{*}^{(n)}$. Последовательные образы отрезков $\Delta_{0}^{(n-1)}$ и $\Delta_{0}^{(n)}$ до такого возвращения покрьвают всю окружность без наложений (за исключением концов), составляя ее динамическое разбиение $n$-го ранга

$$
\mathbf{P}_{n}=\left\{T^{i} \Delta_{0}^{(n-1)}, 0 \leqslant i<q_{n}\right\} \cup\left\{T^{i} \Delta_{0}^{(n)}, 0 \leqslant i<q_{n-1}\right\} .
$$

Конщы же отрезков из $\mathbf{P}_{n}$ составляют можество

$$
\Xi_{n}=\left\{\xi_{i}, 0 \leqslant i<q_{n-1}+q_{n}\right\}
$$

Назовем $n$-й ренормализачией, $n \geqslant 1$, гомеоморфизма $T$ с иррациональным числом вращения относительно отмеченной точки $\xi_{0} \in \mathbb{S}^{1}$ пару функций $f_{n}:[-1,0] \rightarrow \mathbb{R}$, 
$g_{n}:\left[0, a^{(n)}\right] \rightarrow \mathbb{R}, a^{(n)}=f_{n}(0)$, полученных из ограничений $T^{q_{n}}$ на $\Delta_{0}^{(n-1)}$ и $T^{q_{n-1}}$ на $\Delta_{0}^{(n)}$ соответственно с помощью аффинной замены координат, переводящей $\xi_{0}$ в 0 , а $\xi_{q_{n-1}}$ в -1 . Заметим, что $a^{(n)}=f_{n}(0)$ есть просто перенормированная координата точки $\xi_{q_{n}}$. Из нашего определения автоматически следует, что $f_{n}$ и $g_{n}$ строго возрастают, причем график $f_{n}$ лежит строго выше графика тождественного отображения отрезка $[-1,0]$ в себя.

Определенную только что последовательность ренормализаций можно получить также другим способом, а именно итерируя определяемое ниже преобразование, действующее в пространстве пар интервальных отображений, отвечающих гомеоморфизмам окружности. Обозначим $\mathfrak{S}$ множество всех пар функций $F:[-1,0] \rightarrow \mathbb{R}$ и $G:[0, F(0)] \rightarrow \mathbb{R}$, непрерывных, строго возрастающих и удовлетворяющих следуюшим условиям самосогласования: $-1 \leqslant F(-1)<0, F(0) \geqslant 0, G(0)=-1, G(F(0))=$ $F(-1)$. Пару $(F, G) \in \mathfrak{S}$ назовем перенормируемой, если $F^{k}(-1) \leqslant 0<F^{k+1}(-1)$ для некоторого натурального числа $k=k(F, G)$, назьваемого порядком ренормализаций. Легко видеть, что перенормируемьми являются пары, для которых $F$ не имеет неподвижных точек на отрезке $[-1,0]$. Обозначим $\mathfrak{S}_{\mathrm{r}}$ множество всех перенормируемых пар из S. Ренормализационный оператор на парах (далее просто "ренорм-оператор" или "ренорм-преобразование") $\mathscr{R}: \mathfrak{S}_{\mathrm{r}} \rightarrow \mathfrak{S}$ определим равенством $\mathscr{R}(F, G)=\left(F_{1}, G_{1}\right)$, где

$$
\begin{aligned}
& F_{1}(t)=-(F(0))^{-1} F^{k}(G(-F(0) t)), \quad t \in[-1,0] ; \\
& G_{1}(t)=-(F(0))^{-1} F(-F(0) t), \quad t \in\left[0, F_{1}(0)\right],
\end{aligned}
$$

a $k$ - порядок ренормализации для пары $(F, G) \in \mathfrak{S}_{\mathrm{r}}$.

Пару $(F, G)$ назовем бесконечно перенормируемой, если $\mathscr{R}^{n}(F, G) \in \mathfrak{S}_{\mathrm{r}}$ для любого $n \geqslant 0$. Нетрудно убедиться, что ренормализации $\left(f_{n}, g_{n}\right)$, отвечающие гомеоморфизму $T$ с иррациональним числом вращения, бесконечно перенормируемы, причем последовательность ренормализаций образует траекторию ренорм-преобразования:

$$
\mathscr{R}\left(f_{n}, g_{n}\right)=\left(f_{n+1}, g_{n+1}\right) .
$$

Для пары $(F, G) \in \mathfrak{S}$ можно определить число вращения $\rho(F, G) \in[0,1)$. При этом последовательность порядков ренормализации используется в качестве неполных частных в разложении $\rho(F, G)$ в цепную дробь: $\rho(F, G)=\left[k_{1}, k_{2}, \ldots\right]$, где $k_{n}=k\left(\mathscr{R}^{n-1}(F, G)\right)$. Разложение продолжается, пока $\mathscr{R}^{n-1}(F, G) \in \mathfrak{S}_{\mathrm{r}}$, и поэтому может оказаться пустым, конечным либо бесконечным, доставляя для $\rho(F, G)$ нулевое, рациональное или иррациональное значение соответственно. Поскольку преобразование Гаусса $\mathscr{G}$ действует на цепных дробях как единичный сдвиг влево: $\mathscr{G}\left[k_{1}, k_{2}, \ldots, k_{n}, \ldots\right]=\left[k_{2}, k_{3}, \ldots, k_{n}, \ldots\right]$, мы имеем очевидное соотношение $\rho(\mathscr{R}(F, G))=\mathscr{G} \rho(F, G)$, вьполненное для всех перенормируемых пар. Отметим, что понятие числа вращения для пар $(F, G)$ имеет следующий геометрический смысл. Всякая пара $(F, G) \in \mathfrak{S}$ определяет гомеоморфизм $T_{F, G}$ окружности, получаемой из отрезка $[-1, F(0)]$ с отождествленньми концами. Нетрудно убедиться, что число врашения для $T_{F, G}$ равняется $\rho\left(T_{F, G}\right)=\left[k_{1}+1, k_{2}, \ldots, k_{n}, \ldots\right]$, где $k_{i}$ - порядки ренормализаций. (Лишняя единица в первом неполном частном связана с особенностями используемой схемы ренормализации и не играет сушественной роли в дальнейшем.)

Таким образом, приведенная конструкция позволяет рассматривать последовательность ренормализаций $\left(f_{n}, g_{n}\right)$, построенных по гомеоморфизму $T$, как траекторию бесконечномерной динамической системы $(\mathfrak{S} ; \mathscr{R})$, порождаемой действием 
ренорм-оператора в пространстве бесконечно перенормируемых самосогласованных пар. В следующем разделе мы покажем, как могут быть исследованы гиперболические свойства этой динамической системы в случае пространства пар, отвечающих особенностям типа излома.

Отметим, что $(\mathfrak{S} ; \mathscr{R})$ не является динамической системой в классическом смысле, поскольку $\mathscr{R}$ определено не на всем $\mathfrak{S}$. При этом траектории точек в нашей системе могут быть конечными, обрываясь при попадании в $\mathfrak{S} \backslash \mathfrak{S}_{\mathrm{r}}$ (в случае иррациональных чисел врашения). Тем не менее, классические понятия теории (инвариантное множество, аттрактор и пр.) легко обобщаются на данный тип систем. В следующем разделе мы говорим о поглощающих областях и аттракторе типа "подковы" для ренормализаций, отвечаюших диффеоморфизмам с изломами.

\section{3. Диффеоморфизмы с изломами}

Гомеоморфизм окружности $T$ назовем диффеоморфизмом гладкости $C^{2+\alpha}, \alpha \in$ $(0,1)$, с изломом в точке $\xi_{0}$, если для любого его поднятия $L$ выполнены следуюшие условия:

1) $L \in C^{2+\alpha}\left(\left(\xi_{0}, 1+\xi_{0}\right)\right)$;

2) $\inf _{y \in(0,1)} L^{\prime}(y)>0$;

3) существуют односторонние производные $L^{\prime}\left(\xi_{0}+\right) \neq L^{\prime}\left(\xi_{0}-\right)$.

Величиной излома такого диффеоморфизма будем называть число $c=\sqrt{\frac{L^{\prime}\left(\xi_{0}-\right)}{L^{\prime}\left(\xi_{0}+\right)}}$, которое в силу определения положительно и отлично от единицы.

Множеству диффеоморфизмов гладкости $C^{2+\alpha}$ с изломами соответствует множество пар $(F, G) \in \mathfrak{S}$, обе компоненты которых обладают $C^{2+\alpha}$-гладкостью и положительностью производных на своих (замкнутых!) областях определения, и при этом вьполнено условие $c^{2}=\frac{F^{\prime}(0) G^{\prime}(F(0))}{G^{\prime}(0) F^{\prime}(-1)}$. Обозначим пространство таких пар $\mathfrak{S}^{2+\alpha, c} \subset \mathfrak{S}$. Ренорм-оператор $\mathscr{R}$ отображает $\mathfrak{S}^{2+\alpha, c} \cap \mathfrak{S}_{\mathrm{r}}$ в $\mathfrak{S}^{2+\alpha, 1 / c}$. (Переход от $c$ к $1 / c$ связан с изменением ориентации на каждом шаге ренормализации.) Таким образом, $\mathfrak{S}^{2+\alpha, c}$ инвариантно относительно $\mathscr{R}^{2}$ в том смысле, что дважды ренормализуемые пары из $\mathfrak{S}^{2+\alpha, c}$ остаются в $\mathfrak{S}^{2+\alpha, c}$ под действием $\mathscr{R}^{2}$. Для диффеоморфизма с изломом величины $c$ его ренормализации $\left(f_{n}, g_{n}\right)$, определенные в предыдушем разделе, принадлежат множеству $\mathfrak{S}^{2+\alpha, c}$ при четных значениях $n$ и множеству $\mathfrak{S}^{2+\alpha, 1 / c}$ при нечетных.

Рассмотрим семейство пар дробно-линейных функций вида

$$
F_{a, v, c}(z)=\frac{a+c z}{1-v z}, \quad G_{a, v, c}(z)=\frac{a(z-c)}{a c+z(1+v-c)} .
$$

Это семейство играет исключительно важную роль в теории диффеоморфизмов с изломами, поскольку ренормализации $\left(f_{n}, g_{n}\right)$ таких диффеоморфизмов экспоненщиально быстро приближаются к семейству пар вида (2) в пределе при $n \rightarrow+\infty$. Более точно, справедливо следующее утверждение. Пусть $T$ - произвольньй диффеоморфизм гладкости $C^{2+\alpha}$ с изломом величины $c$ и с иррациональным числом вращения. Положим $c^{(n)}=c$ для четных $n$ и $c^{(n)}=1 / c$ для нечетных. Обозначим

$$
a^{(n)}=\frac{\left|\xi_{q_{n}}-\xi_{0}\right|}{\left|\xi_{q_{n-1}}-\xi_{0}\right|}, \quad b^{(n)}=\frac{\left|\xi_{q_{n}+q_{n-1}}-\xi_{0}\right|}{\left|\xi_{q_{n-1}}-\xi_{0}\right|}, \quad v^{(n)}=\frac{c^{(n)}-a^{(n)}-b^{(n)}}{b^{(n)}} .
$$


УТВЕРЖДЕНИЕ 1. Существуют константы $C=C(T)>0, \lambda=\lambda(T) \in(0,1)$ такие, что для любого $n \geqslant 1$

$$
\left.\left\|f_{n}-F_{a^{(n)}, v^{(n)}, c^{(n)}}\right\|_{C^{2+\alpha}} \leqslant C \lambda^{n}, \quad \| g_{n}-G_{a^{(n)}, v^{(n)}, c^{(n)}}\right) \|_{C^{2+\alpha}} \leqslant \frac{C \lambda^{n}}{\left(a^{(n)}\right)^{1+\alpha}} .
$$

Формально утверждение 1 было доказано в [25] в случае $C^{2}$-нормы, однако его перенесение на случай $C^{2+\alpha}$-топологии не представляет труда. Поскольку ренормализации сходятся к семейству (2), мы в дальнейшем изучаем действие ренорм-оператора для пар, отвечаюших этому семейству.

Нетрудно убедиться, что пары $\left(F_{a, v, c}, G_{a, v, c}\right)$ принадлежат $\mathfrak{S}^{2+\alpha, c}$ в том и только том случае, когда параметры $(a, v)$ удовлетворяют условиям

$$
0<a<c, v>c-a-1 \text { либо } a=0, v=c-1 .
$$

Для фиксированного $c$ обозначим полученное подмножество $\mathfrak{L}_{c} \subset \mathfrak{S}^{2+\alpha, c}$. Поскольку $\mathscr{R}\left(\mathfrak{L}_{c} \cap \mathfrak{S}_{\mathrm{r}}\right) \subset \mathfrak{L}_{1 / c}$, то динамика, задаваемая преобразованием $\mathscr{R}$ на $\mathfrak{L}_{c}$ и $\mathfrak{L}_{1 / c}$, является, по сушеству, двумерной. А именно, отождествив пары функций из $\mathfrak{L}_{c}$ с соответствующими точками $(a, v, c)$ в пространстве параметров, нетрудно проверить, что для перенормируемых пар действие ренорм-оператора $\mathscr{R}(a, v, c)=\left(a^{\prime}, v^{\prime}, c^{\prime}\right)$ задается следуюшими соотношениями:

$$
a^{\prime}=-a^{-1} F_{a, v, c}^{k}(-1), \quad v^{\prime}=c^{-1}\left(1-v F_{a, v, c}^{k}(-1)\right)-1, \quad c^{\prime}=1 / c,
$$

где $k$ - порядок ренормализации для $\left(F_{a, v, c}, G_{a, v, c}\right)$. Обозначим $\mathscr{R}_{c}(a, v)=\left(a^{\prime}, v^{\prime}\right)$ преобразование, отвечающее $\mathscr{R}$ при фиксированном $c$. Мы будем также использовать обозначение $\mathscr{R}_{c}^{2}=\mathscr{R}_{1 / c} \circ \mathscr{R}_{c}$. Преобразование $\mathscr{R}_{c}$ определено только для перенормируемых точек $(a, v)$, множество которых мы обозначим $\mathfrak{L}_{\mathrm{r}}^{c}=\{(a, v):(a, v, c) \in$ $\left.\mathfrak{L}_{c} \cap \mathfrak{S}_{\mathrm{r}}\right\}$. Простое вычисление дает $\mathfrak{L}_{\mathrm{r}}^{c}=\{0<a<c, v+a>c-1\}$ для $0<c<1$ и $\mathfrak{L}_{\mathrm{r}}^{c}=\{0<a<c, v+a>c-1\} \backslash\left\{2 v>c-1,4 a v \leqslant(c-1)^{2}\right\}$ для $c>1$. Определим также двумерные области $\mathfrak{D}^{c}=\{(a, v): 1 / 2<v /(c-1)<1, c(c-v-1) / v<a<c\}$, играющие роль поглошающих множеств для динамики, задаваемой ренорм-оператором.

УТВЕРЖДЕНИЕ 2 (поглощение). Для всякого положительного $c \neq 1$ область $\mathfrak{D}^{c}$ инвариантна относительно $\mathscr{R}_{c}^{2}$, причем $\mathscr{R}_{c}\left(\mathfrak{D}^{c} \cap \mathfrak{L}_{r}^{c}\right) \subset \mathfrak{D}^{1 / c}$. Кроме того, траектория произвольной точки $(a, v, c) \in \mathfrak{L}_{c} c$ иррациональныцм числом вращения в некоторый момент попадает в $\mathfrak{D}^{c} \times\{c\}$ и уже не может покинуть $\left(\mathfrak{D}^{c} \times\{c\}\right) \cup\left(\mathfrak{D}^{1 / c} \times\{1 / c\}\right)$.

Таким образом, $\mathfrak{D}^{c}$ есть (ограниченная в $\mathbb{R}^{2}$ ) инвариантная поглощающая область в системе с $\left(\left\{(a, v):(a, v, c) \in \mathfrak{L}_{c}\right\} ; \mathscr{R}_{c}^{2}\right)$, и динамика оператора $\mathscr{R}_{c}^{2}$ на $\mathfrak{D}^{c}$ определяет предельное поведение всех бесконечных траекторий, а значит - и интересующие нас предельные свойства ренормализаций произвольных диффеоморфизмов с изломами.

Обозначим $\mathfrak{D}=\left\{(a, v, c):(a, v) \in \mathfrak{D}^{c}, c>0, c \neq 1\right\}$. Оказьвается, что ренормализационное преобразование $\mathscr{R}$ симметрично обратимо на $\mathfrak{D}$, т.е. в этой области существует обратное преобразование $\mathscr{R}^{-1}$, инволютивно сопряженное с $\mathscr{R}$. Прежде всего определим инволющию $\mathscr{I}(a, v, c)=\left(a^{\prime}, v^{\prime}, c^{\prime}\right)$ и соответствуюшие ей при фиксированном $c$ преобразования $\mathscr{I}_{c}(a, v)=\left(a^{\prime}, v^{\prime}\right)$ соотношениями

$$
a^{\prime}=\frac{c-1-v}{a v}, \quad v^{\prime}=-\frac{v}{c}, \quad c^{\prime}=\frac{1}{c} .
$$

Нетрудно проверить, что $\mathscr{I}$ действительно инволютивно $\left(\mathscr{I}^{2}=\mathrm{Id}\right)$ и что $\mathscr{I}_{c}$ отображает $\mathfrak{D}^{c}$ на $\mathfrak{D}^{1 / c}$. 
УТВЕРЖДЕНИЕ 3 (обратимость). Преобразование $\mathscr{R}$ отображает множество $\mathfrak{D} \cap \mathfrak{S}_{\mathrm{r}}$ на его образ взаимно однозначно. Инволюиия $\mathscr{I}$ переводит $\mathfrak{D} \cap \mathfrak{S}_{\mathrm{r}} u$ $\mathscr{R}\left(\mathfrak{D} \cap \mathfrak{S}_{\mathrm{r}}\right)$ друг в друга. При этом $\mathscr{I}$ в области $\mathfrak{D}$ сопрягает $\mathscr{R}$ с обратным. $\mathscr{I} \circ \mathscr{R}^{-1}=\mathscr{R} \circ \mathscr{I}$, дде обе части равенства определены на $\mathscr{R}\left(\mathfrak{D} \cap \mathfrak{S}_{\mathrm{r}}\right)$.

Смысл утверждения 3 состоит в следуюшем. Вообше говоря, для точки $(a, v, c) \in \mathfrak{D}$ имеется бесконечно много прообразов относительно $\mathscr{R}$ в $\mathfrak{S}$, и только один из них лежит в $\mathfrak{D}$. Однако, поскольку $\mathfrak{D}$ является поглощающей областью, именно этот прообраз нас и интересует. Таким образом, на $\mathfrak{D}$ можно однозначно определить $\mathscr{R}^{-1}$. Замечательное обстоятельство состоит в том, что оператор “деренормализации" $\mathscr{R}^{-1}$ инволютивно сопряжен с ренорм-оператором $\mathscr{R}$, причем инволюция может быть указана явно. Эта симметрия позволяет для любой точки $(a, v, c) \in \mathfrak{D}$ определить ее обобщенное число вращения. Обозначим $\rho(a, v, c)=\rho\left(F_{a, v, c}, G_{a, v, c}\right)$, где $\rho(F, G)$ было определено в предыдушем разделе. Обобшенным числом врашения назовем пару $\left(\rho^{-}(a, v, c), \rho^{+}(a, v, c)\right)$, где $\rho^{+}(a, v, c)=\rho(a, v, c)$, а $\rho^{-}(a, v, c)=\rho(\mathscr{I}(a, v, c))$. Условимся назьвать $\rho^{-}(a, v, c)$ и $\rho^{+}(a, v, c)$ числами врашения назад и вперед соответственно. Для нас особый интерес представляет ситуация, в которой оба числа врашения являются иррациональными, т.е. обе пары $\left(F_{a, v, c}, G_{a, v, c}\right)$ и $\left(F_{\mathscr{I}(a, v, c)}, G_{\mathscr{I}(a, v, c)}\right)$ являются бесконечно перенормируемыми. В этом случае для точки $(a, v, c) \in \mathfrak{D}$ можно однозначно определить ее двустороннюю траекторию $\left(a_{n}, v_{n}, c_{n}\right)=\mathscr{R}^{n}\left(a_{0}, v_{0}, c_{0}\right) \in \mathfrak{D}, n=0, \pm 1, \pm 2, \ldots$ (для $n<0$ мы имеем $\mathscr{R}^{n}=\mathscr{I} \circ \mathscr{R}^{|n|} \circ \mathscr{I}$ в соответствии с утверждением 3$)$. При этом обобщенное число вращения $\rho^{ \pm}(a, v, c)$ удобно записать в виде бесконечной в обе стороны последовательности натуральных чисел $\rho^{ \pm}(a, v, c)=\left[\ldots, k_{-2}, k_{-1}, k_{0}, k_{1}, k_{2}, \ldots\right]$, отвечающей разложению $\rho^{-}(a, v, c)$ и $\rho^{+}(a, v, c)$ в цепные дроби: $\rho^{-}(a, v, c)=$ $\left[k_{0}, k_{-1}, k_{-2}, \ldots\right], \rho^{+}(a, v, c)=\rho(a, v, c)=\left[k_{1}, k_{2}, \ldots\right]$. Нетрудно убедиться, что ренорм-преобразование $\mathscr{R}$ отвечает единичному сдвигу двусторонней последовательности $\left[\ldots, k_{-2}, k_{-1}, k_{0}, k_{1}, k_{2}, \ldots\right]$ влево, а $\mathscr{R}^{-1}$ - вправо. В таких обозначениях особенно хорошо видно, что в области $\mathfrak{D}$ не происходит "потери информации" при ренормализации (имеющей место в $\mathfrak{S})$.

Как показано в [28], для произвольного фиксированного $c \neq 1$ точки $(a, v, c) \in \mathfrak{L}_{c} \mathrm{c}$ иррациональншм числом врашения $\rho(a, v, c)=\left[k_{1}, k_{2}, \ldots\right]$ образуют в $(a, v)$-плоскости грабик $\left\{a=a_{\rho, c}(v), v>-1\right\}$, в то время как точкам с рациональным числом вращения $\rho(a, v, c)=p / q$ отвечает полоса ненулевой ширины $\left\{a_{p / q, c}^{(1)}(v) \leqslant a \leqslant a_{p / q, c}^{(2)}(v)\right.$, $v>-1\}$. При этом все кривые $a_{\rho, c}(v), a_{\rho, c}^{(1)}(v), a_{\rho, c}^{(2)}(v)$ непрерьвны и упорядочены по $\rho$. Из симметрии, задаваемой инволюцией $\mathscr{I}$, следует наличие в $\mathfrak{D}^{c}$ также и структуры, дуальной к описанной в [28], состоящей из кривых и полос, отвечаюших иррациональньм и рациональньм числам вращения назад $\rho^{-}$. (Мы назьваем эти две структуры дуальными, поскольку они переводятся друг в друга инволюцией $\mathscr{I}$.)

Действие $\mathscr{R}_{c}$ на $\mathfrak{D}^{c}$ для всякого $c \neq 1$ может быть описано следующим образом (рис. 1,2$)$. Из треугольной области $\mathfrak{D}^{c}$ нарезается последовательность четырехугольных "полосок" $\Pi_{c, k}^{+}=\left\{(a, v) \in \mathfrak{D}^{c}: 1 /(k+1) \leqslant \rho^{+}(a, v, c)<1 / k\right\}, k \geqslant 1$, состоящих из точек с одним и тем же порядком ренормализации $k$ (на каждой такой полоске оператор $\mathscr{R}_{c}$ непрерывен). В случае $c>1$ от $\mathfrak{D}_{c}$ остается "уголок" из неперенормируемых точек $\left\{(a, v) \in \mathfrak{D}^{c}: \rho(a, v, c)=0\right\}$ (рис. $\left.1 \mathrm{a}\right) ;$ при $c<1$ таких точек в $\mathfrak{D}_{c}$ нет, и вся область расслаивается на полоски $\Pi_{c, k}^{+}$(рис. 2a). Каждая полоска $\Pi_{c, k}^{+}$ под действием $\mathscr{R}_{c}$ деформируется (с изменением ориентации) в треугольньй “лом- 
тик" $\Pi_{1 / c, k}^{-}=\left\{(a, v) \in \mathfrak{D}^{1 / c}: 1 /(k+1) \leqslant \rho^{-}(a, v, c)<1 / k\right\}$ с тем же $k \geqslant 1$, вытянутьй вдоль оси $a$, и полученные ломтики последовательно подклеиваются друг к другу встьк, составляя вместе область $\mathfrak{D}_{1 / \text { c }}$ без “уголка" из лишенных прообраза точек $\left\{(a, v) \in \mathfrak{D}^{1 / c}: \rho^{-}(a, v, 1 / c)=0\right\}$ в случае $c>1$ (рис. $\left.1 \mathrm{~b}\right)$; при $c<1$ таких точек в области $\mathfrak{D}_{1 / c}$ нет, и она заполняется “ломтиками” целиком (рис. $2 \mathrm{~b}$ ).

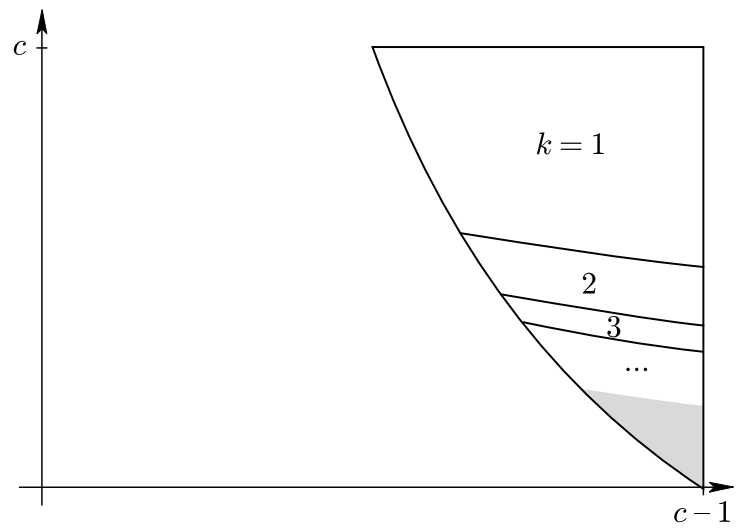

Рис. 1a.

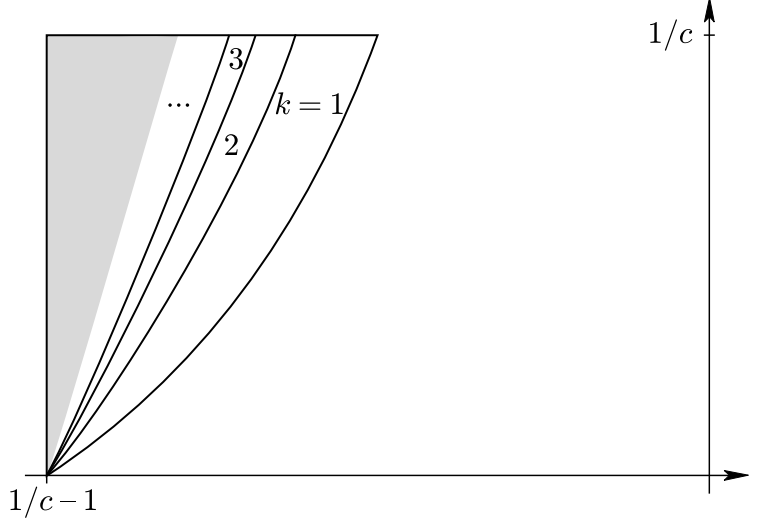

Рис. 1b.

Схема действия оператора $\mathscr{R}_{c}$ на области $\mathfrak{D}^{c}$ при $c>1:$ a) "полоски" $\Pi_{c, k}^{+}$для $k=$ $1,2,3, \ldots$ в $\left.\mathfrak{D}^{c} ; \mathrm{b}\right)$ их образы в $\mathfrak{D}^{1 / c_{-}}$“ломтики” $\Pi_{c, k}^{-}$. (Координата $v$ отложена по горизонтали, $a$ по вертикали; "уголок" неренормализуемых точек в $\mathfrak{D}^{c}$ закрашен; значение $c$ взято равным 4.)

Оказьвается, что преобразование $\mathscr{R}_{c}^{2}$ на $\mathfrak{D}^{c}(c \neq 1$ фиксировано) обладает ярко выраженньми гиперболическими свойствами. А именно, оно сжимает область вдоль семейства кривых $\left\{a_{\rho, c}(v)\right\}$ и растягивает вдоль трансверсального к нему семейства $\mathscr{I}_{1 / c}\left(\left\{a_{\rho, 1 / c}(v)\right\}\right)$. Для количественного описания гиперболичности удобно использо- 


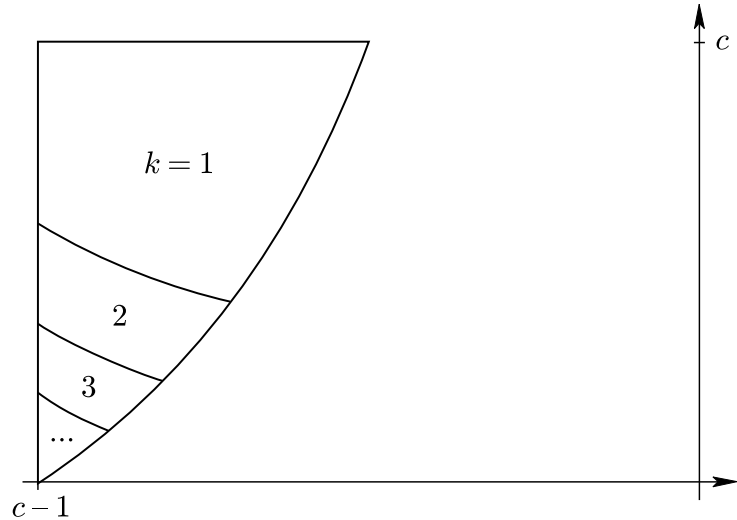

Рис. 2a.

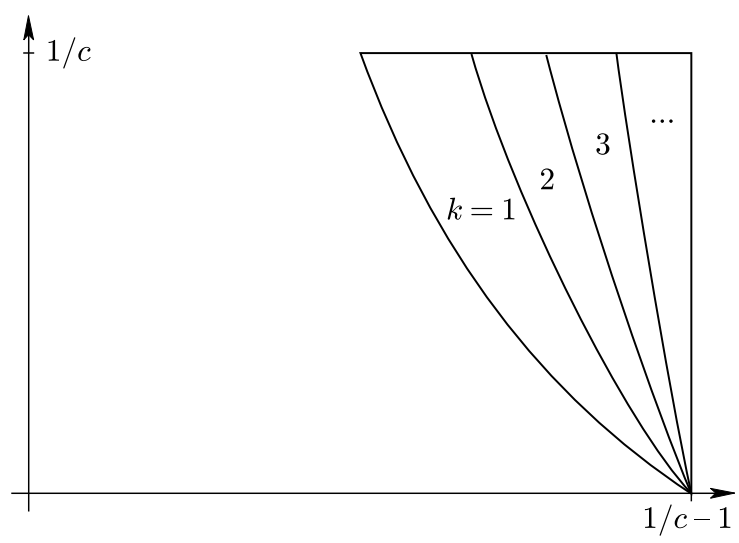

Рис. 2b.

То же, что на рис. 1 , но при $c<1$. ("Уголок" точек без прообраза в $\mathfrak{D}^{1 / c}$ закрашен; значение $c$ взято равным $1 / 4$, так что области $\mathfrak{D}^{c}$ и $\mathfrak{D}^{1 / c}$ на рис. 2 совпадают соответственно с областями $\mathfrak{D}^{1 / c}$ и $\mathfrak{D}^{c}$ на рис. 1.)

вать следующую специальную метрику:

$$
\mathrm{d}_{c}[(a, v),(\widetilde{a}, \widetilde{v})]=|a v-\widetilde{a} \widetilde{v}|+\left|\frac{v+1-c}{c a}-\frac{\widetilde{v}+1-c}{c \widetilde{a}}\right|
$$

для $(a, v),(\widetilde{a}, \widetilde{v}) \in \mathfrak{D}^{c}$. Отметим, что эта метрика не слабее стандартной в $C^{2+\alpha}([-1,0])$ в том смысле, что для любых двух точек $(a, v),(\widetilde{a}, \widetilde{v}) \in \mathfrak{D}^{c}$

$$
\left\|F_{a, v, c}-F_{\widetilde{a}, \widetilde{v}, c}\right\|_{C^{2+\alpha}} \leqslant A \mathrm{~d}_{c}[(a, v),(\widetilde{a}, \widetilde{v})]
$$

с некоторой константой $A=A_{c, \alpha}>0$. Следующее утверждение играет центральную роль в исследовании гиперболических свойств ренорм-преобразования $\mathscr{R}$.

УТВЕРЖДЕНИЕ 4 (гиперболичность). Существуют такие положительные константы $\left\{B_{c}\right\}_{c \neq 1}$, что для любих двух точек $(a, v, c),(\widetilde{a}, \widetilde{v}, c) \in \mathfrak{D} \cap \mathfrak{S}_{\mathrm{r}}$ с одним и тем же иррачиональным числом вращения выполнено неравенство

$$
\mathrm{d}_{1 / c}\left[\mathscr{R}^{c}(a, v), \mathscr{R}^{c}(\widetilde{a}, \widetilde{v})\right] \leqslant B_{c} \mathrm{~d}_{c}[(a, v),(\widetilde{a}, \widetilde{v})] ;
$$


при этом $B_{c} B_{1 / c}<1$.

Утверждение 4 означает, что для всякого $c \neq 1$ оператор $\mathscr{R}_{c}^{2}$ равномерно гиперболичен на $\mathfrak{D}_{c}$. Кривые $\left\{(a, v): a=a_{\rho, c}(v)\right\}_{\rho \notin \mathbb{Q}}$ являются для него устойчивыми, а $\mathscr{I}_{1 / c}\left\{(a, v): a=a_{\rho, 1 / c}(v)\right\}_{\rho \notin \mathbb{Q}}-$ неустойчивыми инвариантными многообразиями. Множество, состоящее из точек пересечения устойчивых и неустойчивых семейств, совпадает со множеством точек, для которых разложение обобщенного числа врашения в цепную дробь бесконечно в обе стороны. Замькание $\mathfrak{A}_{c}$ этого множества представляет из себя канторовское множество нулевой меры - хаотический аттрактор системы $\left(\mathfrak{D}^{c} ; \mathscr{R}_{c}^{2}\right)$ типа “подковы Смейла" с классической символической динамикой на нем.

\section{4. Сопряжения диффеоморфизмов с особенностями}

В этом разделе мы сформулируем и обсудим доказательство Условной теоремы, являюшейся основньм результатом работы. Теоремы 1 и 2 являются ее сравнительно простыми следствиями. Говоря здесь о диффеоморфизмах с особенностями, мы каждый раз подразумеваем диффеоморфизмы окружности гладкости $C^{2+\alpha}$ с одной особой точкой, которая может быть или критической, или точкой излома.

4.1. Критерий гладкости сопряжения. Если для трех гомеоморфизмов окружности $T, \widetilde{T}$ и $h$ выполнено соотношение

$$
\widetilde{T} \circ h=h \circ T,
$$

то говорят, что $T$ и $\widetilde{T}$ сопряжены посредством гомеоморфизма $h$, назьваемого в этом случае сопряжением гомеоморфизмов $T$ и $\widetilde{T}$. Легко показать, что сопряжение $h$ сохраняет число врашения $\rho(T)=\rho(\widetilde{T})$. Если $\widetilde{\xi}_{0}=h\left(\xi_{0}\right)$ является начальной точкой траектории гомеоморфизма $\widetilde{T}$, то сопряжение $h$ переводит все $\xi_{i}=T^{i} \xi_{0}, i \geqslant 0$, в соответствующие точки траектории $\widetilde{\xi}_{i}=\widetilde{T}^{i} \widetilde{\xi}_{0}, i \geqslant 0$, а значит, и элементы динамических разбиений $\mathbf{P}_{n}$ оно отображает в соответствуюшие им элементы $\widetilde{\mathbf{P}}_{n}$. Отметим, что при построении динамических разбиений, рассматриваемых ниже, всегда используется траектория особой точки. Нетрудно видеть, что сопряжение может быть гладким лишш в том случае, когда оно переводит особую точку в особую точку. Именно такие сопряжения мы и рассматриваем ниже.

Сформулируем общий критерий $C^{1}$-гладкости сопряжения $h$ гомеоморфизмов окружности $T$ и $\widetilde{T}$ с совпадающими иррациональными числами вращения, исходящий из близости отношений соответствуюших друг другу элементов двух динамических разбиений. Положим $\sigma(I)=|h(I)| /|I|$ для отрезка $I \subset \mathbb{S}^{1}$, где $|\cdot|$ обозначает длину отрезка.

УТвЕРЖДЕнИЕ 5 (критерий гладкости). Если существуют такие константы $C>0$ и $\mu \in(0,1)$, что, начиная $с$ некоторого момента $n \geqslant n_{0}$, для любых двух әлементов $I, I^{\prime} \in \mathbf{P}_{n+1}$, которые либо имеют общий конец, либо содержатся $в$ одном и том же әлементе динамического разбиения $\mathbf{P}_{n}$, выполнена оценка

$$
\left|\ln \sigma(I)-\ln \sigma\left(I^{\prime}\right)\right| \leqslant C \mu^{n},
$$

то сопряжение $h$ является $C^{1}$-гладким на $\mathbb{S}^{1}$. 
4.2. Условная теорема. Пусть $T$ и $\widetilde{T}$ - два диффеоморфизма окружности с особенностями в точках $\xi_{0}$ и $\widetilde{\xi}_{0}$, а $f_{n}$ и $\widetilde{f}_{n}-$ первые компоненты их $n$-х ренормализаций относительно отмеченных точек $\xi_{0}$ и $\widetilde{\xi}_{0}$ соответственно, определенные в разделе 2 . (Далее мы назьваем $f_{n}$ и $\tilde{f}_{n}$ просто ренормализациями.)

Теорема 3 (условная теорема). Предположим, что для Т и Т выполнены следующие условия:

1) тип особенности совпадает для обоих диффеоморфизмов, т.е. или обе точ$\kappa и \xi_{0}$ и $\widetilde{\xi}_{0}$ являются критическими одного порядка $1+\beta, \beta>0$, или же обе являются точками излома с одинаковой величиной излома $0<c \neq 1$;

2) числа вращения также совпадают и иррациональны: $\rho(T)=\rho(\widetilde{T})=\rho \notin \mathbb{Q}$, причем в случае излома дополнительно предполагается, что $\rho \in M_{\mathrm{o}}$ в случае $c>1$ и что $\rho \in M_{\mathrm{e}}$ в случае $c<1$;

3) существуют такие константы $S>0 u \lambda \in(0,1)$, что для всех $n$ верна оченка

$$
\left\|f_{n}-\widetilde{f}_{n}\right\|_{C^{2+\alpha}} \leqslant S \lambda^{n}
$$

Тогда сопряжение $h$ диффеоморфизмов с особенностями $T$ и $\widetilde{T}$ является $C^{1}$ гладким.

Чтобы доказать теорему 3 , нам необходимо установить выполнение условий утверждения 5. Вьвод необходимой оценки (3) проводится непосредственно для произвольного достаточно большого $n$, однако, уже зафиксировав такое $n$, мы перебираем все требуюшие рассмотрения пары элементов $\left(I, I^{\prime}\right)$ динамического разбиения $\mathbf{P}_{n+1}$, начав вблизи особой точки и постепенно удаляясь от нее в соответствии со следующей логической схемой.

Вначале неравенство вида (3) доказьвается для тех элементов $I$ и $I^{\prime}$, что содержатся в множестве $\Delta_{*}^{(n)}$, представляюшем из себя минимальную окрестность особой точки, составленную из элементов разбиения $\mathbf{P}_{n}$. Именно рассмотрение этого крайнего случая, несмотря на его комбинаторную простоту, является центральным моментом во всем доказательстве; именно на этом шаге выявляются и преодолеваются основные сложности, связанные с появлением почти параболических точек в случае атипичных чисел вращения. Этот Этап доказательства, как наиболее содержательньй в идейном плане, мы ниже объясняем подробнее.

Второй этап рассуждений представляет из себя индукционный переход, позволяюший распространить оценку вида $(3)$ с множества $\Delta_{*}^{(m)}$ на $\Delta_{*}^{(m-1)}$ для $m \leqslant n$. Здесь необходимо подробно отследить комбинаторику траекторий (описьваемую арифметическими свойствами цепных дробей), с тем чтобы указать для каждой “новой" пары $\left(I, I^{\prime}\right)$ отрезков из $\Delta_{*}^{(m-1)}$ такую “старую" пару в $\Delta_{*}^{(m)}$, что в результате применения к обеим ее компонентам определенной последовательности итераций исходного гомеоморфизма окружности старая пара перейдет в новую, причем все эти итерации не сильно испортят интересующую нас оценку вида (3).

К сожалению, начатую индукцию нельзя довести до конца (т.е. до $\Delta_{*}^{(0)}=\mathbb{S}^{1}$ ) ввиду того факта, что с каждым индукционным шагом накапливается погрешность в поддерживаемой нами оценке разности из (3), становясь неудовлетворительно большой, когда число таких шагов приближается к $n$. Но все же, как показьвают вычисления, для достаточно малого числа $\nu>0$ для любого $n \geqslant n_{0}$ первые $[\nu n]$ шагов нашей индукции могут быть проведены с выполнением в итоге неравенства $(3)$ для $I, I^{\prime}$ в $\Delta_{*}^{(n-[\nu n])}$. 
Для диффеоморфизмов с обоими рассматриваемыми нами типами особенности этого оказывается достаточно для того, чтобы известными способами (см. [28] для излома и [19] для критической точки) "разнести” оценку (3) на всю окружность, что и является третьим, заключительным этапом доказательства теоремы 3.

4.3. Регулярность ренормализаций. Ключевым для понимания поведения траекторий диффеоморфизмов с особенностями является свойство равномерной невырожденности их ренормализаций, описываемое нашим следуюшим утверждением.

Напомним, что график $f_{n}$ лежит строго выше диагонали (так мы называем график тождественной функции на $[-1,0]$ ), почти касаясь ее в случае, если $k_{n}$ велико. Говоря ниже о конкретной точке почти касания, мы имеем в виду точку $z_{*}$ максимального приближения $f_{n}$ к тождественной функщии. Такую точку $z_{*}$ мы называем почти параболической, если $f_{n}$ обладает $C^{2+\alpha}$-гладкостью в открытом интервале, содержашем $z_{*}$ (из чего вытекает $f_{n}^{\prime}\left(z_{*}\right)=1$ ), и в нем $f_{n}^{\prime \prime}(z) \geqslant$ const $>0$.

УТВЕРЖДЕНИЕ 6. Для любого диффеоморфизма T, удовлетворяющего условиям на тип особенности и число вращения, налагаемым в теореме 3 , найдется такой набор из четырех положительных констант $K_{1}, K_{2}, K_{3}, K_{4}$, что следующие четыре условия будут выполнень для всякого $n$ :

i) $\left\|f_{n}\right\|_{C^{2+\alpha}} \leqslant K_{1}$;

ii) множсество $M_{f_{n}, K_{2}}=\left\{z \in[-1,0], f_{n}(z)-z<K_{2}\right\}$ либо пусто, либо является открытым интервалом;

iii) $f_{n}^{\prime \prime}(z)>K_{3}$ для любого $z \in M_{f_{n}, K_{2}}$;

iv) $f_{n}^{\prime}(z)>K_{4}$ для любого $z \in\left[-1,-K_{2}^{2}\right]$.

Основной качественньй смысл утверждения 6 состоит в том, что график $f_{n}$ может иметь лишш одну точку почти касания с диагональю и что такая точка обязана быть почти параболической. Именно это свойство отличает рассматриваемые нами дифффеоморфизмы окружности с особенностями от гладких диффеоморфизмов, поскольку в последнем случае при больших $k_{n}$ графики ренормализаций плотно прилегают к диагонали на всем ее протяжении. Дополнительное условие на число вращения, налагаемое теоремой 3 в случае диффеоморфизмов с изломом, позволяет исключить ситуацию, в которой $f_{n}$ вьпукла вверх, а точками почти касания являются концы отрезка $[-1,0]$ (см. [28]).

Доказательство утверждения 6 проводится для диффеоморфизмов с изломом и для критических поворотов по-разному. В первом случае условия i)-iv) могут быть проверены непосредственно [25], а в случае критических поворотов они следуют, например, из отрицательности производной Шварца для $f_{n}$.

Отметим, что из условия іi) вытекает, что $a^{(n)} \geqslant K_{2}$ и что элементы динамического разбиения $\mathbf{P}_{n+1}$, лежашие на отрезке $\Delta_{*}^{(n)}$ ближе к его концам, имеют длину того же порядка, что и $\Delta_{*}^{(n)}$ (в частности, оба крайних элемента имеют длину, не меньшую $\left.K_{2}\left|\Delta_{0}^{(n-1)}\right|\right)$. Кроме того, условие iv) гарантирует, что $f_{n}^{\prime}(z)>K_{4}$ для всех $z$, отвечаюших точкам окружности, лежашим на отрезке с концами в $\xi_{q_{n-1}}$ и $\xi_{q_{n+1}}$.

4.4. Главный этап доказательства. В этом подразделе мы обсуждаем доказательство оценки (3) для элементов $I$ и $I^{\prime}$ разбиения $\mathbf{P}_{n+1}$, содержашихся в отрезке $\Delta_{*}^{(n)}$. Все константы предполагаются не зависящими от шага ренормализации $n$.

4.4.1. Начальные построения. Из утверждения 6 и условия 3) теоремы 3 следует, что $\left|\ln \sigma\left(\Delta_{0}^{(n-1)}\right)-\ln \sigma\left(\Delta_{0}^{(n)}\right)\right|=\left|\ln f_{n}(0)-\ln \widetilde{f}_{n}(0)\right| \leqslant C_{1} \lambda^{n}$ для $n \geqslant n_{0}$ 
с некоторой константой $C_{1}>0$. Отсюда вытекает существование предела $r=$ $\lim _{n \rightarrow+\infty} \ln \sigma\left(\Delta_{0}^{(n)}\right) \in \mathbb{R}$, а также экспоненциальная по $n$ (с основанием $\lambda$ ) скорость сходимости к нему. Мы можем добиться равенства $r=0$, подправив $T$ или $\widetilde{T}$ с помошью бесконечно гладкого диффеоморфизма. Действительно, в случае $r<0$ мы заменим $T$ на $\phi \circ T \circ \phi^{-1}$, где $\phi-$ сохраняюший ориенташию диффеоморфизм окружности $\mathbb{S}^{1}$ гладкости $C^{\infty}$, аффинньй на $\Delta_{*}^{(2)}$ с коэффициентом растяжения $e^{r}<1$. Такая деформация диффеоморфизма $T$ не отразится ни на его ренормализациях $f_{n}$, $n \geqslant 1$, ни на типе особенности, ни на числе врашения, при том что значение предела $r$ станет равным нулю. В случае же $r>0$ аналогичньм образом следует деформировать $\widetilde{T}$.

Итак, в дальнейшем мы будем предполагать, что пара диффеоморфизмов $T$ и $\widetilde{T}$ уже подверглась описанной вьше процедуре, так что для любого $n \geqslant n_{0}$ имеет место оценка

$$
\left|\ln \sigma\left(\Delta_{0}^{(n)}\right)\right| \leqslant C_{2} \lambda^{n}
$$

с положительной константой $C_{2}$.

Обозначим $I_{0}^{(n)}=T^{q_{n-1}} \Delta_{0}^{(n)}$ и $I_{i}^{(n)}=T^{q_{n}} I_{i-1}^{(n)}$ для $i \geqslant 1$. Легко убедиться в том, что отрезками $I_{i}^{(n)}, 0 \leqslant i<k_{n}, \Delta_{0}^{(n+1)}$ и $\Delta_{0}^{(n)}$ исчерпьваются все элементы разбиения $\mathbf{P}_{n+1}$, содержашиеся в отрезке $\Delta_{*}^{(n)}$ (причем они примыкают один к другому именно в том порядке, в котором были только что выписаны).

УТВЕРЖДЕНИЕ 7. Пусть выполнены условия теоремы 3. Найдутся такие константы $C_{3}>0 u \mu \in(0,1)$, что для любых $n \geqslant n_{0} u 0 \leqslant i<k_{n}$ будет иметь место оченка

$$
\left|\ln \sigma\left(I_{i}^{(n)}\right)\right| \leqslant C_{3} \mu^{n}
$$

Вместе с (4) утверждение 7 обеспечивает вьполнение оценки (3) для $I, I^{\prime} \subset \Delta_{*}^{(n)}$, в доказательстве чего и состоит первый и наиболее важный этап доказательства теоремы 3. Заметим, что оценка (5) справедлива равномерно по значениям $k_{n}$.

В перенормированных координатах

$$
\sigma\left(I_{i}^{(n)}\right)=\frac{\left|z_{i+1}-z_{i}\right|}{\left|\widetilde{z}_{i+1}-\widetilde{z}_{i}\right|},
$$

где $z_{0}=\widetilde{z}_{0}=-1$ и $z_{i+1}=f_{n}\left(z_{i}\right), \widetilde{z}_{i+1}=f_{n}\left(\widetilde{z}_{i}\right)$ для $0 \leqslant i<k_{n}$ (в целях упрощения обозначений мы не наделяем переменные $z$ и $\widetilde{z}$ необходимыми им по смыслу индексами $n)$.

Мы будем последовательно шагать по точкам отрезка траектории $z_{i}=f_{n}^{i}(-1)$, $0 \leqslant i \leqslant k_{n}$, стартовав сразу от обоих его краев, $z_{0}=-1$ и $z_{k_{n}}=-a^{(n+1)} a^{(n)}$, от первого - вправо, а от второго - влево. Оба встречных движения сойдутся вблизи почти параболической точки. Каждое из них последовательно пройдет четыре условных стадии (части рассматриваемого отрезка траектории), анализируемых нами по-разному. В нижеследуюшем описании стадий мы предполагаем $k_{n}$ достаточно большим (растушим быстрее чем экспоненциально по $n$ ). В случае, когда $k_{n}$ не столь велико, доказательство утверждения 7 просто заканчивается раньше - на одном из своих промежуточных шагов.

4.4.2. Первая стадия - макроскопическая. Эта стадия содержит конечное (не зависяшее от $n$ ) число шагов. А именно, отсчитаем ровно по $l=\left[1 / K_{2}\right]+1$ шагов от обоих краев. Справедливо следуюшее утверждение. 
Лемма 1. Существует такая константа $C_{4}>0$, что

$$
\left|z_{i}-\widetilde{z}_{i}\right| \leqslant C_{4} \lambda^{n}
$$

для $0 \leqslant i \leqslant l+1$ u для $k_{n}-l-1 \leqslant i \leqslant k_{n}$.

Доказательство этой леммы не вызывает сложностей в силу утверждения 6 и условия 3) теоремы 3 . Из леммы 1 следует оценка (5) с $\mu=\lambda$ для $i \leqslant l$ и для $i \geqslant k_{n}-l-1$, поскольку, как уже указывалось, в силу того же утверждения 6 положительные величины $z_{i+1}-z_{i}$ и $\widetilde{z}_{i+1}-\widetilde{z}_{i}$ (длины перенормированных отрезков $I_{i}^{(n)}$ ) для таких $i$ отделены от нуля не зависящей от $n$ константой.

Как несложно заметить, для всех оставшихся значений $i$-точнее, для $l \leqslant i \leqslant k_{n}-l$ - точки $z_{i}$ содержатся внутри интервала $M_{f_{n}, K_{2}} \neq \varnothing$, который мы называем "воронкой" и где $f_{n}$ строго выпукла вниз.

4.4.3. Вторая стадия - спуск в воронку. Вторая стадия состоит из экспоненциального по $n$ числа шагов. Следуюшие две леммы справедливы вдоль всего рассматриваемого отрезка траектории $z_{i}, 0 \leqslant i \leqslant k_{n}$.

ЛЕмма 2. Существует такая константа $C_{5}>0$, что

$$
\left|z_{i}-\widetilde{z}_{i}\right| \leqslant C_{5} \lambda^{n / 2}
$$

для $0 \leqslant i \leqslant k_{n}$.

Для $l \leqslant i \leqslant\left[\lambda^{-n / 2}\right]$ оценка (6) доказьвается посредством итерирования неравенства

$$
\left|z_{i+1}-\widetilde{z}_{i+1}\right| \leqslant\left|f_{n}\left(z_{i}\right)-f_{n}\left(\widetilde{z}_{i}\right)\right|+\left|f_{n}\left(\widetilde{z}_{i}\right)-\widetilde{f}_{n}\left(\widetilde{z}_{i}\right)\right| \leqslant\left|z_{i}-\widetilde{z}_{i}\right|+S \lambda^{n}
$$

для $k_{n}-\left[\lambda^{-n / 2}\right] \leqslant i \leqslant k_{n}-l$ необходимо проитерировать неравенство

$$
\left|z_{i-1}-\widetilde{z}_{i-1}\right| \leqslant\left|f_{n}^{-1}\left(z_{i}\right)-f_{n}^{-1}\left(\widetilde{z}_{i}\right)\right|+\left|f_{n}^{-1}\left(\widetilde{z}_{i}\right)-\widetilde{f}_{n}^{-1}\left(\widetilde{z}_{i}\right)\right| \leqslant\left|z_{i}-\widetilde{z}_{i}\right|+C_{6} \lambda^{n},
$$

верное с некоторой константой $C_{6}>0$; для остальных значений $i$ оценка (6) вытекает просто из близости всех точек $z_{i}, \widetilde{z}_{i}, z_{*}$ и $\widetilde{z}_{*}$ друг к другу. (Здесь $z_{*}$ и $\widetilde{z}_{*}-$ единственные почти параболические точки $f_{n}$ и $\widetilde{f}_{n}$ соответственно.)

Лемма 3. Существует такая константа $C_{7}>0$, что

$$
\left|\ln \sigma\left(I_{i+1}\right)-\ln \sigma\left(I_{i}\right)\right| \leqslant C_{7} \lambda^{n / 4}
$$

для $0 \leqslant i \leqslant k_{n}-2$.

Оценка (7) вьводится из леммы 2 по-разному в двух случаях. Если $z_{i+1}-z_{i}>\lambda^{n / 4}$, то дело решает неравенство $\left|\frac{\left|\widetilde{z}_{i+1}-\widetilde{z}_{i}\right|}{\left|z_{i+1}-z_{i}\right|}-1\right| \leqslant \frac{\left|z_{i+1}-\widetilde{z}_{i+1}\right|+\left|z_{i}-\widetilde{z}_{i}\right|}{\left|z_{i+1}-z_{i}\right|}$; в противном же случае достаточно заметить, что $\frac{\left|z_{i+2}-z_{i+1}\right|}{\left|z_{i+1}-z_{i}\right|}=f_{n}^{\prime}(\theta)$ с $\theta \in\left[z_{i}, z_{i+1}\right]$ и что $|\theta-\widetilde{\theta}| \leqslant\left(z_{i+1}-z_{i}\right)+\left|z_{i}-\widetilde{z}_{i}\right|+\left(\widetilde{z}_{i+1}-\widetilde{z}_{i}\right)$.

Из леммы 3 нетрудно вьвести оценку (5) с $\mu=\lambda^{1 / 8}$ для $i \leqslant\left[\lambda^{n / 8}\right]$ и для $i \geqslant k_{n}-$ $\left[\lambda^{n / 8}\right]$ 
4.4.4. Третья стадия - воронка. Аффинная замена координат

$$
x=\psi_{n}(z)=\left(\frac{1}{2} f_{n}^{\prime \prime}\left(z_{*}\right)\right)\left(z-z_{*}\right)
$$

переводит $f_{n}$ в функцию $\Phi_{n}=\psi_{n} f_{n} \psi_{n}^{-1}$, для которой

$$
\Phi_{n}(x)=\varepsilon_{n}+x+x^{2}+O\left(|x|^{2+\alpha}\right),
$$

где $0<\varepsilon_{n}=\Phi_{n}(0) \ll K_{2}$ - “эффективный” диаметр воронки в точке почти касания $x_{*}=\psi_{n} z_{*}=0$. Положим $x_{i}=\psi_{n} z_{i}$.

Доказательство следующего общего утверждения носит технический характер.

ЛЕмма 4 (асимптотика в воронке). Предположим, что последовательность действительных чисел $s_{i}, i \geqslant 0$, удовлетворяет условиям $\left|s_{i+1}-\left(s_{i}-s_{i}^{2}\right)\right| \leqslant$ $S_{1}\left|s_{i}\right|^{2+\alpha}$ с некоторыми константами $S_{1}>0 u \alpha \in(0,1)$. Тогда существуют такие константы $D_{1}=D_{1}\left(\alpha, S_{1}\right)>0 u \tau=\tau\left(\alpha, S_{1}\right) \in(0,1)$, что в случае $s_{0} \in(0, \tau]$ неравенство

$$
\left|s_{i}-\frac{1}{i+s_{0}^{-1}}\right| \leqslant \frac{D_{1}}{\left(i+s_{0}^{-1}\right)^{1+\alpha}}
$$

верно для всех $i \geqslant 0$.

При этом существует такая константа $D_{2}=D_{2}\left(\alpha, S_{1}\right)>0$, что в случае $s_{0} \in(0, \tau]$ оченка

$$
s_{i}-s_{i+1}=\frac{1}{\left(i+s_{0}^{-1}\right)^{2}}\left(1+\delta_{i}\right), \quad\left|\delta_{i}\right| \leqslant D_{2} s_{0}^{\alpha}
$$

верна для всех $i \geqslant 0$.

Асимптотическая формула (8) позволяет применить лемму 4 к последовательностям $s_{i}=-x_{i+\left[\lambda^{n / 8}\right]}, i \geqslant 0$, и $s_{i}=x_{k_{n}-\left[\lambda^{n / 8}\right]-i}, i \geqslant 0$, но только лишь в той части воронки, где возможно пренебречь слагаемым $\varepsilon_{n}$ в правой части (8). Используя (9), мы получаем неравенство (5) с $\mu=\lambda^{\alpha / 8}$ для $i$ таких, что соответствующие им величины $x_{i}$ находятся вне окрестности нуля порядка $\varepsilon_{n}^{\frac{1}{2+\alpha}}$.

Вычисления показьвают, что $\varepsilon_{n} \sim \pi^{2} k_{n}^{-2}$ при $k_{n} \rightarrow+\infty$ и что количество шагов на третьей стадии имеет порядок $k_{n}^{\frac{2}{2+\alpha}}$.

4.4.5. Четвертая стадия - туннель. В последней, наиболее узкой, части воронки, назьваемой нами “туннелем", где лемма 4 неприменима, мы вьнуждены использовать иную асимптотику. Туннель, хотя и представляет из себя очень малую (порядка $\varepsilon_{n}^{\frac{1}{2+\alpha}}$ ) окрестность почти параболической точки $x_{*}=0$, содержит тем не менее основную массу точек рассматриваемого отрезка траектории, т.е. число шагов в туннеле асимптотически (при $\left.k_{n} \rightarrow \infty\right)$ равно обшему числу шагов $k_{n}$. 
ЛЕмма 5 (асимптотика в туннеле). Предположим, что последовательность действительных чисел $s_{i}, i \geqslant 0$, удовлетворяет условиям

1) $\left|s_{0}\right| \leqslant S_{1} \varepsilon$

2) $\left|s_{i+1}-\left(\varepsilon+s_{i}+s_{i}^{2}\right)\right| \leqslant S_{2}\left|s_{i}\right|^{2+\alpha} \partial \Omega_{я} i \geqslant 0$

с некоторыми константами $S_{1}, S_{2}>0$ u $\alpha, \varepsilon \in(0,1)$. Пусть $a_{0} \in[0, \pi / 2)-$ такое число, что $s_{0}=\sqrt{\varepsilon} \operatorname{tg} a_{0}$. Для любого $S_{3}>0$ существуют константь $D_{3}=D_{3}\left(\alpha, S_{1}, S_{2}, S_{3}\right)>0 u \tau=\tau\left(\alpha, S_{1}, S_{2}, S_{3}\right) \in(0,1)$ такие, что для любого $\varepsilon \in(0, \tau]$ и для всех $i \in\left[0, \frac{\pi}{2 \sqrt{\varepsilon}}\right)$, удовлетворяющих оченке

$$
\sqrt{\varepsilon} \operatorname{tg} \sqrt{\varepsilon} i \leqslant S_{3} \varepsilon^{\frac{1}{2+\alpha}}
$$

справедливо неравенство

$$
\left|s_{i}-\sqrt{\varepsilon} \operatorname{tg}\left(\sqrt{\varepsilon} i+a_{0}\right)\right| \leqslant D_{3}(\sqrt{\varepsilon} \operatorname{tg} \sqrt{\varepsilon} i)^{1+\frac{\alpha(\alpha+1)}{2}} .
$$

При этом существует такая константа $D_{4}=D_{4}\left(\alpha, S_{1}, S_{2}, S_{3}\right)>0$, что для любого $\varepsilon \in(0, \tau]$ оценка

$$
s_{i+1}-s_{i}=\frac{\varepsilon}{(\cos \sqrt{\varepsilon} i)^{2}}\left(1+\delta_{i}\right), \quad\left|\delta_{i}\right| \leqslant D_{4} \varepsilon^{\frac{\alpha(\alpha+1)}{2(\alpha+2)}},
$$

верна для всех $i \in\left[0, \frac{\pi}{2 \sqrt{\varepsilon}}\right)$, удовлетворяющих (10).

Применим лемму к последовательностям $s_{i}=x_{i+i_{\mathrm{c}}}, 0 \leqslant i \leqslant\left[\frac{1}{\sqrt{\varepsilon_{n}}} \operatorname{arctg} \frac{\varepsilon_{n}^{1 /(2+\alpha)}}{\sqrt{\varepsilon_{n}}}\right]$, и $s_{i}=\varepsilon_{n}-x_{i_{\mathrm{c}}-i}, 0 \leqslant i \leqslant\left[\frac{1}{\sqrt{\varepsilon_{n}}} \operatorname{arctg} \frac{\varepsilon_{n}^{1 /(2+\alpha)}}{\sqrt{\varepsilon_{n}}}\right]$, где $x_{i_{\mathrm{c}}} \in\left[0, \varepsilon_{n}\right)$. Используя $(9)$, можно наконец получить неравенство (5) с $\mu=\lambda^{\frac{\alpha(1+\alpha)}{16(2+\alpha)}}$ для всех $0 \leqslant i<k_{n}$, доказав тем самым утверждение 7 . Отметим, что значение индекса $\widetilde{i}_{\mathrm{c}}$, получаемое для $\widetilde{T}$, вообще говоря, не совпадает с $i_{\mathrm{c}}$, так что для обоих диффеоморфизмов последовательности в туннеле оказьваются слегка сдвинутыми друг относительно друга, и это требует некоторых дополнительных оценок, на которых мы здесь не останавливаемся. Укажем лишь, что порядок сдвига не превышает $k_{n}^{1-\alpha}$ шагов.

\section{5. Заключение}

В этом разделе мы кратко обсуждаем основные результаты работы и формулируем общую гипотезу о жесткости для диффеоморфизмов окружности с несколькими особыми точками.

В работе изучается теория жесткости для диффеоморфизмов окружности с особенностями. Жесткость означает, что комбинаторика траекторий, задаваемая иррациональным числом вращения, плюс локальная характеристика особенности определяют такой диффеоморфизм с точностью до $C^{1}$-гладкого сопряжения. Иными словами, асимптотические метрические свойства динамических систем, индуцируемых на окружности диффеоморфизмами с одной особой точкой заданного типа и с заданным иррациональным числом вращения, совпадают. Мы показали, что сформулированньй вьше результат справедлив для аналитических критических поворотов окружности с критической точкой нечетного порядка. Замечательно, что жесткость в этом 
случае распространяется на все иррациональные числа врашения (а не только на типичные, как в случае гладких диффеоморфизмов). Следует ожидать, что результат о $C^{1}$-жесткости для всех чисел врашения справедлив для $C^{2+\epsilon}$-гладких критических поворотов с критической точкой произвольного порядка, хотя в настояшее время этот факт не доказан. Заметим, что в случае диффеоморфизмов с одной особой точкой гладкое сопряжение всегда единственно, поскольку оно обязательно переводит особую точку одного диффеоморфизма в особую точку другого.

Мы также рассматриваем диффеоморфизмы окружности с изломом. Для них мы доказываем глобальную гиперболичность оператора ренормализации, почти явно при этом проводя многие построения так называемой Программы Ланфорда. Заметим, что неожиданные симметрии, выявленные нами в этой связи, говорят, по-видимому, о наличии за ренормализационными конструкциями скрытой алгебро-геометрической структуры. Выявление и исследование такой структуры представляется важной и интересной задачей.

Более сильная жесткость в случае диффеоморфизмов с особенностями объясняется тем, что их ренормализации являются функциями, выпуклыми в окрестности почти параболических точек, в которых их графики почти касаются графиика тождественного отображения. Именно благодаря выпуклости динамика вблизи таких точек, возникающих в случае нетипичных чисел вращения, носит в данном случае достаточно регулярный характер. Доказательство жесткости основано на тщательном анализе этой динамики.

В случае отображений с изломами, в отличие от критическим поворотов, мынакладьваем некоторые условия на числа вращения. А именно, мы требуем ограниченности нечетных неполных частных в случае $c>1$ и четных в случае $c<1$, где $c$ - величина излома. Эти ограничения связаны со следуюшим обстоятельством. Нетрудно показать, что для диффеоморфизмов с изломами точки почти касания не всегда являются параболическими. Ровно в половине случаев сама точка излома и является точкой почти касания. Такие ситуации требуют специального изучения. Хотя мы и не занимались подобным анализом, нам представляется правдоподобным, что и в случае излома жесткость верна для всех иррациональных чисел вращения.

Одним из основных этапов исследования в теории жесткости является анализ гиперболических свойств ренормализационного оператора. K настоящему моменту такой анализ проведен в двух случаях: критические повороты нечетного порядка и дифофеоморфизмыс изломами. В обоих случаях доказательства весьма трудны и требуют специальных конструкций. Для критических поворотов существуюший подход к доказательству требует нечетности порядка критической точки. С другой стороны, сам факт, о котором здесь идет речь, представляется весьма и весьма общим. А именно, если два диффеоморфизма имеют особые точки одного типа (произвольного) и одинаковые числа вращения, то их ренормализации экспоненщиально быстро сближаются. Концептуальное доказательство этого факта является центральной задачей теории жесткости. По нашему мнению, эта задача не является безнадежной. При этом имеются основания ожидать, что ее окончательное решение будет получено с помошью действительных, а не комплексно-аналитических методов.

В заключение мы сформулируем достаточно общую гипотезу о жесткости в случае диффеоморфизмов с конечным числом особых точек. Поскольку гладкое сопряжение переводит особую точку данного типа в особую точку того же типа, мы потребуем, чтобы два диффеоморфизма $T$ и $\widetilde{T}$ имели одинаковое число особых точек $z^{1}<z^{2}<\cdots<z^{k}<z^{1}+1$ и $\widetilde{z}^{1}<\widetilde{z}^{2}<\cdots<\widetilde{z}^{k}<\widetilde{z}^{1}+1$, причем для каждого 
$1 \leqslant i \leqslant k$ особые точки $z^{i}$ и $\widetilde{z}^{i}$ имели одинаковьй тип, т.е. либо обе являлись критическими точками одинакового порядка, либо же обе - точками излома с одинаковой величиной излома. В случае, если все особые точки являются точками излома, мы дополнительно потребуем, чтобы произведение изломов не равнялось 1 . Предположим также, что $T$ и $\widetilde{T}$ имеют одинаковое иррациональное число врашения. Обозначим $\mu$ и $\widetilde{\mu}$ единственные нормированные инвариантные меры для $T$ и $\widetilde{T}$ соответственно. Легко видеть, что условие $\mu\left(\left[z^{i}, z^{i+1}\right]\right)=\widetilde{\mu}\left(\left[\widetilde{z}^{i}, \widetilde{z}^{i+1}\right]\right), 1 \leqslant i<k$, является необходимым для существования гладкого сопряжения между $T$ и $\widetilde{T}$. Следующая гипотеза утверждает, что оно является также и достаточньм.

ГИПоТЕЗА 2. Диффеоморфизмы $T$ и $\widetilde{T} C^{1}$-гладко сопряжень между собой тогда и только тогда, когда $\mu\left(\left[z^{i}, z^{i+1}\right]\right)=\widetilde{\mu}\left(\left[\widetilde{z}^{i}, \widetilde{z}^{i+1}\right]\right)$ для всех $1 \leqslant i<k$.

Заметим, что имеется связь между сформулированной вьше гипотезой и работой Р. Мак-Кая [32]. Наконец, отметим еше один класс диффеоморфизмов с особенностями, для которых исключительно важно построить теорию жесткости. Речь идет о диффеоморфизмах, отвечающих критическим инвариантным кривым теории КАМ. Строгие результаты в этой области почти отсутствуют. Из данных численного счета следует, что, по-видимому, возникаюшие таким образом диффеоморфизмы принадлежат классу гладкости $C^{1+\nu}, 0 \leqslant \nu \leqslant 1$. Хотя структура особенностей в этом случае не ясна, нет сомнений, что имеется бесконечное множество особых точек, плотно расположенных на окружности. Таким образом, наша гипотеза не покрывает эту ситуацию. Тем не менее, из нестрогой теории ренормализаций, развитой Мак-Каем, следует, что имеется жесткость для диффеоморфизмов, отвечающих критическим кривым с одним и тем же типичным иррациональньм числом вращения. Отметим, что рассмотрение произвольных иррациональных чисел врашения в этом случае лишено смысла, поскольку само существование инвариантных кривых теории КАМ требует вьполнения некоторых диофантовых условий.

\section{СПИСОК ЛИТЕРАТУРЫ}

[1] А. Н. Колмогоров. О сохранении условно периодических движений при малом изменении функции Гамильтона // Докл. АН СССР. 1954. Т. 98. № 4. С. 527-530.

[2] В. И. Арнольд. Малые знаменатели и проблемы устойчивости движения в классической и небесной механике // УМН. 1963. Т. 18. №6. С. 91-192.

[3] А. Н. Колмогоров. Локальная структура турбулентности в несжимаемой вязкой жидкости при очень больших числах Рейнольдса // Докл. АН СССР. 1941. Т. 30. №4. C. 299-303.

[4] А. Н. Колмогоров. К вырождению изотропной турбулентности в несжимаемой вязкой жидкости // Докл. АН СССР. 1941. Т. 31. №6. С. 538-541.

[5] А.Н. Колмогоров. Рассеяние энергии при локально изотропной турбулентности // Докл. АН СССР. 1941. Т. 32. № 1. С. 19-21.

[6] В.И. Арнольд. Малые знаменатели. І. Об отображениях окружности на себя // Изв. АН CСCP. Сер. матем. 1961. Т. 25. № 1. C. 21-86.

[7] M.-R. Herman. Sur la conjugaison différentiable des difféomorphismes du cercle à des rotations // Inst. Hautes Études Sci. Publ. Math. 1979. № 49. P. 5-233.

[8] M.-R. Herman. Résultats récents sur la conjugaison différentiable // Proceedings of the International Congress of Mathematicians (Helsinki, 1978). Helsinki: Acad. Sci. Fennica, 1980. P. $811-820$.

[9] J.-C. Yoccoz. Conjugaison différentiable des difféomorphismes du cercle dont le nombre de rotation vérifie une condition diophantienne // Ann. Sci. École Norm. Sup. (4). 1984. V. 17. № 3. P. 333-359. 
[10] K. M. Khanin, Ya. G. Sinai. A new proof of M. Herman's theorem // Comm. Math. Phys. 1987. V. 112. № 1. P. 89-101.

[11] Я.Г. Синай, К. М. Ханин. Гладкость сопряжений диффеоморфизмов окружности с поворотами // УМН. 1989. Т. 44. № 1. С. 57-82.

[12] Y. Katznelson, D. Ornstein. The differentiability of the conjugation of certain diffeomorphisms of the circle // Ergodic Theory Dynam. Systems. 1989. V. 9. № 4. P. 643-680.

[13] Y. Katznelson, D. Ornstein. The absolute continuity of the conjugation of certain diffeomorphisms of the circle // Ergodic Theory Dynam. Systems. 1989. V. 9. №4. P. 681-690.

[14] M. J. Feigenbaum. Quantitative universality for a class of nonlinear transformations // J. Statist. Phys. 1978. V. 19. № 1. P. 25-52.

[15] M. J. Feigenbaum. The transition to aperiodic behavior in turbulent systems // Comm. Math. Phys. 1980. V. 77. №1. P. 65-86.

[16] D. Sullivan. Bounds, quadratic differentials, and renormalization conjectures // American Mathematical Society Centennial Publications. V. II. Providence, RI: Amer. Math. Soc., 1992. P. 417-466.

[17] C.T. McMullen. Complex Dynamics and Renormalization. Princeton, NJ: Princeton Univ. Press, 1994. (Ann of Math. Stud. V. 135.)

[18] M. Lyubich. Feigenbaum-Coullet-Tresser universality and Milnor's hairiness conjecture // Ann. of Math. (2). 1999. V. 149. № 2. P. 319-420.

[19] E. de Faria, W. de Melo. Rigidity of critical circle mappings. I. // J. Eur. Math. Soc. (JEMS). 1999. V. 1. № 4. P. 339-392.

[20] E. de Faria, W. de Melo. Rigidity of critical circle mappings. II. // J. Amer. Math. Soc. 2000. V. 13. № 2. P. 343-370.

[21] M. Yampolsky. The attractor of renormalization and rigidity of towers of critical circle maps // Comm. Math. Phys. 2001. V. 218. №3. P. 537-568.

[22] M. Yampolsky. Renormalization horseshoe for critical circle maps // Comm. Math. Phys. 2003. V. 240. № 1-2. P. 75-96.

[23] D. Khmelev, M. Yampolsky. The rigidity problem for analytic critical circle maps // Preprint. Toronto: Univ. of Toronto, 2003.

[24] Е. Б. Вул, К. М. Ханин. Гомеоморфизмы окружности с особенностью типа излома // УМH. 1990. Т. 45. № 3. С. 189-190.

[25] K. M. Khanin, E. B. Vul. Circle homeomorphisms with weak discontinuities // Dynamical Systems and Statistical Mechanics (Moscow, 1991). Providence, RI: Amer. Math. Soc., 1991. P. 57-98. (Adv. Soviet Math. V. 3.)

[26] А. А. Джалилов, К. М. Ханин. Об инвариантной мере для гомеоморфизмов окружности с одним изломом // Функц. анализ и прил. 1998. Т. 32. № 3. С. 11-21.

[27] К. М. Ханин. Жесткость для гомеоморфизмов окружности с особенностью типа излома // Докл. РАН. 1997. Т. 357. № 2. С. 176-179.

[28] K. Khanin, D. Khmelev. Renormalizations and rigidity theory for circle homeomorphisms with singularities of the break type // Comm. Math. Phys. 2003. V. 235. № 1. P. 69-124.

[29] O. E. Lanford. Renormalization group methods for circle mappings / / Nonlinear Evolution and Chaotic Phenomena. New York: Plenum, 1988. P. 25-36. (NATO Adv. Sci. Inst. Ser. B Phys. V. 176.)

[30] K. Khanin, D. Khmelev, A. Teplinsky. Uniform hyperbolicity for renormalizations of circle diffeomorphisms with breaks (to appear).

[31] K. Khanin, A. Teplinsky. Smoothness of conjugacy for circle diffeomorphisms with singularities (to appear).

[32] R.S. MacKay. Renormalisation of bicritical circle maps // Phys. Lett. A. 1994. V. 187. № 5-6. P. 391-396.

Институт математики НАН Украины, Киев;

Поступила в редакцию

Isaac Newton Institute for Mathematical Sciences, Cambridge,

19.06 .2003

Heriot-Watt University, Edinburgh,

Институт теоретической физики им. Л. Д. Ландау, Москва 